\title{
Forbidden triples generating a finite set of 3-connected graphs
}

\author{
Yoshimi Egawa \\ Dept. of Mathematical Information Science \\ Tokyo University of Science \\ Tokyo 162-8601, Japan \\ Michitaka Furuya $^{\dagger}$ \\ Dept. of Mathematical Information Science \\ Tokyo University of Science \\ Tokyo 162-8601, Japan \\ michitaka.furuya@gmail.com
}

\author{
Jun Fujisawa* \\ Faculty of Business and Commerce \\ Keio University \\ Kanagawa 223-8521, Japan \\ fujisawa@fbc.keio.ac.jp \\ Michael D. Plummer \\ Dept. of Mathematics \\ Vanderbilt University \\ Nashville, Tennessee 37240, U.S.A \\ michael.d.plummer@vanderbilt.edu
}

\author{
Akira Saito \\ Dept. of Information Science \\ Nihon University \\ Tokyo 156-8550, Japan \\ asaito@chs.nihon-u.ac.jp
}

Submitted: Mar 31, 2013; Accepted: Jun 28, 2015; Published: Jul 17, 2015

Mathematics Subject Classifications: 05C75

\begin{abstract}
For a graph $G$ and a set $\mathcal{F}$ of connected graphs, $G$ is said be $\mathcal{F}$-free if $G$ does not contain any member of $\mathcal{F}$ as an induced subgraph. We let $\mathcal{G}_{3}(\mathcal{F})$ denote the set of all 3 -connected $\mathcal{F}$-free graphs. This paper is concerned with sets $\mathcal{F}$ of connected graphs such that $|\mathcal{F}|=3$ and $\mathcal{G}_{3}(\mathcal{F})$ is finite. Among other results, we show that for an integer $m \geqslant 3$ and a connected graph $T$ of order greater than or equal to 4 , $\mathcal{G}_{3}\left(\left\{K_{4}, K_{2, m}, T\right\}\right)$ is finite if and only if $T$ is a path of order 4 or 5 .
\end{abstract}

Keywords: forbidden subgraph; forbidden triple; 3-connected graph

\footnotetext{
*Supported by JSPS Grant-in-Aid for Young Scientists (B) (22740068) and Keio Gijuku Academic Development Funds.

†Supported by JSPS Grant-in-Aid for Young Scientists (B) (26800086).

${ }^{\ddagger}$ Partially supposed by JSPS Grant-in-Aid for Scientific Research (C) (22500018).
} 


\section{Introduction}

In this paper, we consider only finite undirected simple graphs.

Let $\mathcal{G}$ denote the set of connected graphs with order greater than or equal to three. For a graph $G$ and for $F \in \mathcal{G}, G$ is said to be $F$-free if $G$ does not contain $F$ as an induced subgraph and, for $\mathcal{F} \subseteq \mathcal{G}, G$ is said to be $\mathcal{F}$-free if $G$ is $F$-free for every $F \in \mathcal{F}$. For an integer $k \geqslant 1$ and $\mathcal{F} \subseteq \mathcal{G}$, we let $\mathcal{G}_{k}$ denote the set of all $k$-connected graphs, and let $\mathcal{G}_{k}(\mathcal{F})$ denote the set of all $\mathcal{F}$-free graphs belonging to $\mathcal{G}_{k}$. Thus

$$
\mathcal{G}_{k}(\mathcal{F}):=\{G \mid G \text { is a } k \text {-connected } \mathcal{F} \text {-free graph }\} .
$$

This paper is concerned with subsets $\mathcal{F}$ of $\mathcal{G}$ such that $\mathcal{G}_{k}(\mathcal{F})$ is a finite set. In this context, members of $\mathcal{F}$ are often referred to as forbidden subgraphs. For detailed historical background and related results, we refer the reader to [3].

The following result can be found in [1]. (Here $K_{n}$ denotes the complete graph of order $n, P_{l}$ denotes the path of order $l$ and, in general, $K_{m_{1}, m_{2}}$ denotes the complete bipartite graph with partite sets having cardinalities $m_{1}$ and $m_{2}$.)

Theorem A (Diestel [1]; Chapter 9). For $\mathcal{F} \subseteq \mathcal{G}, \mathcal{G}_{1}(\mathcal{F})$ is finite if and only if $K_{n}, K_{1, m}$, $P_{l} \in \mathcal{F}$ for some integers $n \geqslant 3, m \geqslant 2$ and $l \geqslant 3$.

For $k \geqslant 2$, it is unlikely that a general result like Theorem A holds. Thus we confine ourselves to the case where $|\mathcal{F}|$ is "small". It is known that for any $k \geqslant 2$, there is no $\mathcal{F} \subseteq \mathcal{G}$ with $|\mathcal{F}|=1$ such that $\mathcal{G}_{k}(\mathcal{F})$ is finite. (See [3]; Theorem 2.) Further, those subsets $\mathcal{F}$ of $\mathcal{G}$ with $|\mathcal{F}|=2$ for which $\mathcal{G}_{k}(\mathcal{F})$ is finite are determined for $k \leqslant 6$ in [3]. Here we are interested in the case where $|\mathcal{F}|=3$. Note that a connected $K_{1,2}$-free graph is a complete graph. Hence if $K_{1,2} \in \mathcal{F}$, then $\mathcal{G}_{k}(\mathcal{F})$ is finite if and only if $K_{n} \in \mathcal{F}$ for some $n \geqslant 3$, and there is no point in forbidding two more graphs. Thus when we discuss $\mathcal{G}_{k}(\mathcal{F})$ with $|\mathcal{F}|=3$, we usually assume $K_{1,2} \notin \mathcal{F}$. For $k=2$, the following theorem is proved in [3].

Theorem B (Fujisawa, Plummer and Saito [3]). Let $\mathcal{F}$ be a subset of $\mathcal{G}$ with $|\mathcal{F}|=3$ and $K_{1,2} \notin \mathcal{F}$. Then $\mathcal{G}_{2}(\mathcal{F})$ is finite if and only if one of the following holds:

(i) $\mathcal{F}=\left\{K_{3}, K_{2, m}, P_{l}\right\}$ for some integers $m$ and $l$ with $m \geqslant 3$ and $4 \leqslant l \leqslant 5$;

(ii) $\mathcal{F}=\left\{K_{3}, K_{2,2}, P_{6}\right\}$; or

(iii) $\mathcal{F}=\left\{K_{n}, K_{1, m}, P_{l}\right\}$ for some integers $n, m$ and $l$ with $n \geqslant 3, m \geqslant 3$ and $l \geqslant 4$.

In the present paper, we investigate the case where $|\mathcal{F}|=3$ and $k=3$. It is easy to see $\mathcal{G}_{3}\left(\left\{K_{3}, K_{1,3}\right\}\right)=\varnothing$. (See [3].) Thus when we consider $\mathcal{G}_{3}(\mathcal{F})$, we assume that $\left\{K_{3}, K_{1,3}\right\} \nsubseteq \mathcal{F}$, in addition to the condition that $K_{1,2} \notin \mathcal{F}$. Before stating our results, we make some more definitions.

Let $n$ be an integer with $n \geqslant 2$. Let $P=x_{1} x_{2} \cdots x_{n}$ be the path of order $n$, and let $y_{1}, y_{2}, z_{1}$ and $z_{2}$ be four distinct vertices different from $x_{1}, \ldots, x_{n}$. We let $Y_{n}, Y_{n}^{*}, Q_{n}$ and $Q_{n}^{*}$ denote the graphs defined by

$$
V\left(Y_{n}\right)=V\left(Q_{n}\right)=V(P) \cup\left\{y_{1}, y_{2}\right\}, \quad V\left(Y_{n}^{*}\right)=V\left(Q_{n}^{*}\right)=V(P) \cup\left\{y_{1}, y_{2}, z_{1}, z_{2}\right\},
$$




$$
\begin{gathered}
E\left(Y_{n}\right)=E(P) \cup\left\{x_{1} y_{1}, x_{1} y_{2}\right\}, \quad E\left(Y_{n}^{*}\right)=E(P) \cup\left\{x_{1} y_{1}, x_{1} y_{2}, x_{n} z_{1}, x_{n} z_{2}\right\}, \\
E\left(Q_{n}\right)=E\left(Y_{n}\right) \cup\left\{y_{1} y_{2}\right\} \quad \text { and } \quad E\left(Q_{n}^{*}\right)=E\left(Y_{n}^{*}\right) \cup\left\{y_{1} y_{2}, z_{1} z_{2}\right\}
\end{gathered}
$$

(see Figure 1).
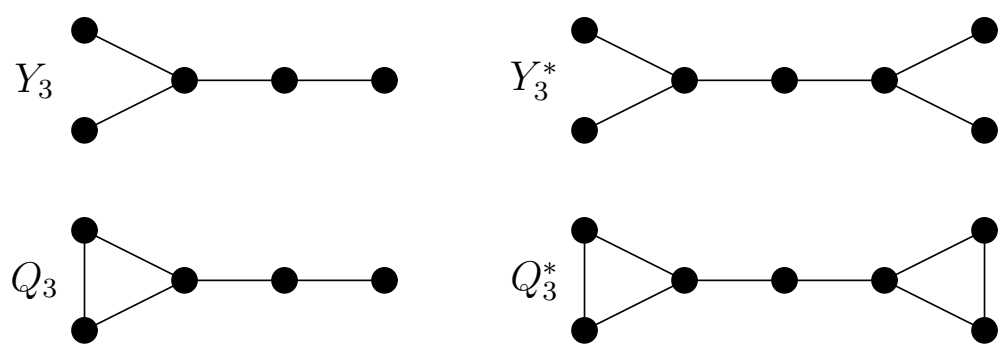

Figure 1: Graphs $Y_{3}, Y_{3}^{*}, Q_{3}$ and $Q_{3}^{*}$

A caterpillar is a tree for which the removal of all endvertices leaves a path. A complete bipartite graph of the form $K_{1, m}$ with $m \geqslant 1$ is called a star. Let $\mathcal{T}_{0}$ be the set of trees in $\mathcal{G}-\left\{K_{1,2}, K_{1,3}\right\}$ having maximum degree at most 3 . Note that $\mathcal{T}_{0}$ does not contain a star. Let $\mathcal{T}_{1}$ be the set of those caterpillars belonging to $\mathcal{T}_{0}$ in which no two vertices of degree 3 are adjacent. Let $\mathcal{T}_{2}=\left\{P_{l}, Y_{m}, Y_{n}^{*} \mid l \geqslant 4, m \geqslant 3, n \geqslant 3\right\}$. We have $\mathcal{T}_{0} \supseteq \mathcal{T}_{1} \supseteq \mathcal{T}_{2}$.

Let $G$ be a connected graph. A vertex $v$ of $G$ is called a cutvertex if $G-v$ is disconnected. If $G$ has a cutvertex, $G$ is said to be separable; otherwise, it is said to be nonseparable. Note that $K_{1}$ is a nonseparable graph. A maximal nonseparable subgraph of $G$ is called a block of $G$. When $G$ is separable, the block-cutvertex graph of $G$ is defined to be the bipartite graph $Z$ such that $Z$ has as its partite sets the set of all cutvertices of $G$ and the set of all blocks of $G$ and, for a cutvertex $v$ and a block $B, v$ and $B$ are adjacent in $Z$ if and only if $v$ is a vertex of $B$ in $G$. It is a well-known fact that the block-cutvertex graph of a connected graph is a tree. A cactus is a connected graph every block of which is a complete graph of order two or a cycle. Let $\mathcal{T}_{0}^{*}$ be the set of those cacti $T$ in $\mathcal{G}-\left\{K_{1,2}, K_{3}\right\}$ such that all cycles of $T$ are triangles and in the block-cutvertex graph of $T$, the distance between any two vertices corresponding to triangles of $T$ is a multiple of 4 . Let $\mathcal{T}_{1}^{*}$ be the set of those members of $\mathcal{T}_{0}^{*}$ whose block-cutvertex graph is a path. Let $\mathcal{T}_{2}^{*}=\left\{P_{l}, Q_{m}, Q_{2 n}^{*} \mid l \geqslant 4, m \geqslant 2, n \geqslant 1\right\}$. We have $\mathcal{T}_{0}^{*} \supseteq \mathcal{T}_{1}^{*} \supseteq \mathcal{T}_{2}^{*}$.

Our main result is as follows.

Theorem 1.1. Let $\mathcal{F}$ be a subset of $\mathcal{G}$ with $|\mathcal{F}|=3, K_{1,2} \notin \mathcal{F}$ and $\left\{K_{1,3}, K_{3}\right\} \not \mathcal{F}$, and suppose that $\mathcal{G}_{3}(\mathcal{F})$ is finite. Then one of the following holds:

(i) $\mathcal{F}=\left\{K_{3}, K_{3, m}, T\right\}$ with $m \geqslant 3$, where $T \in \mathcal{T}_{2}$;

(ii) $\mathcal{F}=\left\{K_{4}, K_{2, m}, T\right\}$ with $m \geqslant 2$, where $T$ is a path;

(iii) $\mathcal{F}=\left\{K_{3}, K_{2, m}, T\right\}$ with $m \geqslant 2$, where $T \in \mathcal{T}_{0}$ in the case where $m=2, T \in \mathcal{T}_{1}$ in the case where $3 \leqslant m \leqslant 4$, and $T \in \mathcal{T}_{2}$ in the case where $m \geqslant 5$; 
(iv) $\mathcal{F}=\left\{K_{n}, K_{1, m}, T\right\}$ with $n \geqslant 4$ and $m \geqslant 4$, where $T$ is a path;

(v) $\mathcal{F}=\left\{K_{3}, K_{1, m}, T\right\}$ with $m \geqslant 4$, where $T \in \mathcal{T}_{1}$ in the case where $m=4$, and $T \in \mathcal{T}_{2}$ in the case where $m \geqslant 5$; or

(vi) $\mathcal{F}=\left\{K_{n}, K_{1,3}, T\right\}$ with $n \geqslant 4$, where $T \in \mathcal{T}_{1}^{*}$ in the case where $n=4$, and $T \in \mathcal{T}_{2}^{*}$ in the case where $n \geqslant 5$.

The converse of Theorem 1.1 does not hold. However, if (iv) of Theorem 1.1 holds, then $\mathcal{G}_{3}(\mathcal{F})$ is finite by Theorem A. Also, as we shall state below in Theorem 1.5, if (v) holds with $m \geqslant 5$, then $\mathcal{G}_{3}(\mathcal{F})$ is finite. Further when $m$ is "large" in cases (i) through (iii) of Theorem 1.1, we can determine $T$ as follows.

Theorem 1.2. Let $m$ be an integer with $m \geqslant 4$, and let $T \in \mathcal{G}-\left\{K_{1,2}, K_{1,3}\right\}$. Then $\mathcal{G}_{3}\left(\left\{K_{3}, K_{3, m}, T\right\}\right)$ is finite if and only if $T$ is a path of order 4 or 5 or $T=Y_{3}$.

Theorem 1.3. Let $m$ be an integer with $m \geqslant 3$, and let $T \in \mathcal{G}-\left\{K_{1,2}\right\}$. Then $\mathcal{G}_{3}\left(\left\{K_{4}, K_{2, m}, T\right\}\right)$ is finite if and only if $T$ is a path of order 4 or 5 .

Theorem 1.4. Let $m$ be an integer with $m \geqslant 5$, and let $T \in \mathcal{G}-\left\{K_{1,2}, K_{1,3}\right\}$. Then $\mathcal{G}_{3}\left(\left\{K_{3}, K_{2, m}, T\right\}\right)$ is finite if and only if $T$ is either a path of order at most 7 or an induced subgraph of $Y_{3}^{*}$.

Theorem 1.5. Let $m$ be an integer with $m \geqslant 5$, and let $T \in \mathcal{G}-\left\{K_{1,2}, K_{1,3}\right\}$. Then $\mathcal{G}_{3}\left(\left\{K_{3}, K_{1, m}, T\right\}\right)$ is finite if and only if $T \in \mathcal{T}_{2}$.

We prove Theorem 1.1 in Section 2. We prove Theorem 1.2 in Section 3, Theorem 1.3 in Section 4, Theorem 1.5 in Section 5, and Theorem 1.4 in Section 6. Our notation and terminology are standard, and mostly taken from [1]. Exceptions are as follows. Let $G$ be a graph. For $u, v \in V(G), d(u, v)$ denotes the distance between $u$ and $v$. When $G$ is connected, we define the diameter $\operatorname{diam}(G)$ of $G$ by $\operatorname{diam}(G)=\max \{d(u, v) \mid u, v \in$ $V(G)\}$. Let $u \in V(G)$. For an integer $i \geqslant 1$, we let $N_{i}(u)=\{x \in V(G) \mid d(u, x)=i\}$. We write $N(u)$ for $N_{1}(u)$. We let $d(u)$ denote the degree of $u$; thus $d(u)=|N(u)|$. When we need to specify that the underlying graph is $G$, we write $N_{G}(u)$ and $d_{G}(u)$ for $N(u)$ and $d(u)$, respectively. We let $\Delta(G)=\max \{d(u) \mid u \in V(G)\}$. For $Y \subseteq V(G)$, we let $N(Y)$ denote the union of $N(u)$ as $u$ ranges over $Y$. For $X, Y \subseteq V(G)$ with $X \cap Y=\varnothing$, $E(X, Y)$ denotes the set of edges joining a vertex in $X$ and a vertex in $Y$. When $G$ is connected, a block of $G$ containing at most one cutvertex of $G$ is called an endblock of $G$. When $G$ is not necessarily connected, by a cutvertex of $G$, we mean a cutvertex of a component of $G$. Similarly, by a block (resp. an endblock) of $G$, we mean a block (resp. an endblock) of a component of $G$. Note that isolated vertices of $G$ are endblocks of $G$. For an endblock $B$ of $G$, a vertex of $B$ which is not a cutvertex of $G$ is called an internal vertex of $B$. For a graph $H$ and an integer $s \geqslant 2$, we let $s H$ denote the disjoint union of $s$ copies of $H$. For two graphs $H_{1}$ and $H_{2}$, we let $H_{1}+H_{2}$ denote the join of $H_{1}$ and $H_{2}$. Finally for $s \geqslant 4, C_{s}$ denotes the cycle of order $s$ and, for $t \geqslant 5$, we let $W_{t}=C_{t-1}+K_{1}$ denote the wheel graph of order $t$. 
In subsequent arguments, when we prove the finiteness of $\mathcal{G}_{3}(\mathcal{F})$ for a given family $\mathcal{F}$, we bound the diameter and the maximum degree of a graph $G$ in $\mathcal{G}_{3}(\mathcal{F})$ from above, and then bound the order in terms of the diameter and the maximum degree. For this purpose, we make one easy observation.

Lemma 1.6. Let $m \geqslant 2$ and $k \geqslant 3$, and let $G$ be a graph with $\Delta(G) \leqslant m$ and $\operatorname{diam}(G) \leqslant$ $k$. Then $|V(G)| \leqslant m^{k}$.

Proof. Let $w \in V(G)$. Then $\left|N_{i}(w)\right| \leqslant m(m-1)^{i-1}$ for each $1 \leqslant i \leqslant k$. Hence $|V(G)| \leqslant$ $1+m+\left(\sum_{2 \leqslant i \leqslant k-1} m(m-1)^{i-1}\right)+m(m-1)^{k-1} \leqslant m^{k-1}+\left(\sum_{2 \leqslant i \leqslant k-1} m^{k-i}(m-1)^{i-1}\right)+$ $m(m-1)^{k-1}=m^{k}$.

The bound $m^{k}$ in the above lemma is far from sharp, but we use it for the sake of brevity.

\section{A necessary condition}

In this section, we prove Theorem 1.1. We start with several lemmas. The first two lemmas are proved in [4] and [3], respectively.

Lemma 2.1 (Kochol [4]). For every integer $g \geqslant 3$, there exists a 3-connected 3-regular graph with girth $g$. In particular, for every integer $g \geqslant 3$, there exist infinitely many 3-connected 3-regular graphs with girth at least $g$.

Lemma 2.2 (Fujisawa, Plummer and Saito [3]). For $\mathcal{F} \subseteq \mathcal{G}$, if $\mathcal{G}_{3}(\mathcal{F})$ is finite, then $\left\{K_{n}, K_{m_{1}, m_{2}}\right\} \subseteq \mathcal{F}$ for some integers $n, m_{1}$ and $m_{2}$ with $n \geqslant 3, m_{2} \geqslant m_{1} \geqslant 1$ and $m_{1} \leqslant 3$.

Lemma 2.3. Let $\mathcal{F}$ be a finite subset of $\mathcal{G}-\left\{K_{1,2}, K_{1,3}\right\}$, and suppose that $\mathcal{G}_{3}(\mathcal{F})$ is finite. Then $\mathcal{F}$ contains a member of $\mathcal{T}_{0}$.

Proof. Let $t=\max \{|V(F)| \mid F \in \mathcal{F}\}$, and let $\mathcal{H}=\left\{G \in \mathcal{G}_{3} \mid G\right.$ is a 3-regular graph with girth at least $t+1\}$. By Lemma $2.1, \mathcal{H}$ is an infinite set. Since $\mathcal{G}_{3}(\mathcal{F})$ is finite and $\mathcal{H}$ is infinite, there exists $G \in \mathcal{H}$ such that $G$ contains a graph $F$ in $\mathcal{F}$ as an induced subgraph. Since the girth of $G$ is strictly greater than $|V(F)|, F$ is a tree. Since $G$ is 3-regular and since $F \neq K_{1,2}, K_{1,3}$ by the assumption that $\mathcal{F} \subseteq \mathcal{G}-\left\{K_{1,2}, K_{1,3}\right\}$, it follows that $F \in \mathcal{T}_{0}$.

Lemma 2.4. Let $m_{1}$ and $m_{2}$ be integers such that $m_{2} \geqslant m_{1} \geqslant 1, m_{1} \leqslant 3$ and $\left(m_{1}, m_{2}\right) \neq$ $(1,2),(1,3),(2,2)$, and let $T \in \mathcal{G}-\left\{K_{1,2}, K_{1,3}\right\}$. Set $\mathcal{F}=\left\{K_{3}, K_{m_{1}, m_{2}}, T\right\}$, and suppose that $\mathcal{G}_{3}(\mathcal{F})$ is finite. Then the following hold.

(i) We have $T \in \mathcal{T}_{1}$.

(ii) If in addition, $\left(m_{1}, m_{2}\right) \notin\{(1,4),(2,3),(2,4)\}$, then $T \in \mathcal{T}_{2}$.

Proof. By Lemma 2.3, $T \in \mathcal{T}_{0}$. 
(i) For each $s \geqslant 5$, the Cartesian product $C_{s} \times K_{2}$ is 3 -connected and $\left\{K_{3}, K_{m_{1}, m_{2}}\right\}$-free. Since $\mathcal{G}_{3}(\mathcal{F})$ is finite, this implies that there exists $s \geqslant 5$ such that $C_{s} \times K_{2}$ contains $T$ as an induced subgraph. Since every member of $\mathcal{T}_{0}$ contained in $C_{s} \times K_{2}$ as an induced subgraph belongs to $\mathcal{T}_{1}, T \in \mathcal{T}_{1}$.

(ii) By (i), $T \in \mathcal{T}_{1}$. For each $s \geqslant 5$, let $C_{s}^{\prime}$ denote the so-called lexicographic product of $C_{s}$ and the null graph of order two; that is to say, $V\left(C_{s}^{\prime}\right)=\left\{x_{i, j} \mid 1 \leqslant i \leqslant s, 1 \leqslant j \leqslant\right.$ $2\}$ and $E\left(C_{s}^{\prime}\right)=\left\{x_{i, j} x_{i+1, h} \mid 1 \leqslant i \leqslant s, 1 \leqslant j, h \leqslant 2\right\}$, where first indices of the letter $x$ are to be read modulo $s$. Then $C_{s}^{\prime}$ is 3 -connected and $\left\{K_{3}, K_{m_{1}, m_{2}}\right\}$-free. Hence there exists $s \geqslant 5$ such that $C_{s}^{\prime}$ contains $T$ as an induced subgraph. Since every member of $\mathcal{T}_{1}$ contained in $C_{s}^{\prime}$ as an induced subgraph belongs to $\mathcal{T}_{2}, T \in \mathcal{T}_{2}$.

Proof of Theorem 1.1. Let $\mathcal{F}$ be as in Theorem 1.1. By Lemma 2.2, $\left\{K_{n}, K_{m_{1}, m_{2}}\right\} \subseteq \mathcal{F}$ for some integers $n, m_{1}$ and $m_{2}$ with $n \geqslant 3, m_{2} \geqslant m_{1} \geqslant 1$ and $m_{1} \leqslant 3$. Write $\mathcal{F}=$ $\left\{K_{n}, K_{m_{1}, m_{2}}, T\right\}$.

Case 1: $\mathcal{F}$ contains no star

In this case, $2 \leqslant m_{1} \leqslant 3$, and we have $T \in \mathcal{T}_{0}$ by Lemma 2.3 .

Subcase 1.1: $m_{1}=3$

For each $s \geqslant 3, P_{3}+s K_{1}$ is 3 -connected and $K_{3, m_{2}}$-free. Since $T$ is not a star and every tree contained in $P_{3}+s K_{1}$ as an induced subgraph is a star, $P_{3}+s K_{1}$ is also $T$-free. Since $\mathcal{G}_{3}(\mathcal{F})$ is finite, this implies that there exists $s \geqslant 3$ such that $P_{3}+s K_{1}$ contains $K_{n}$ as an induced subgraph. Since $P_{3}+s K_{1}$ is $K_{4}$-free, this forces $n=3$. Now by Lemma 2.4(ii), $T \in \mathcal{T}_{2}$, and hence (i) of Theorem 1.1 holds.

Subcase 1.2: $m_{1}=2$ and $n \geqslant 4$

For each $s \geqslant 3, K_{3}+s K_{1}$ is 3 -connected and $K_{2, m_{2}}$-free. Since every tree contained in $K_{3}+s K_{1}$ as an induced subgraph is a star, $K_{3}+s K_{1}$ is also $T$-free. Hence there exists $s \geqslant 3$ such that $K_{3}+s K_{1}$ contains $K_{n}$ as an induced subgraph, which implies $n=4$. For each $t \geqslant 6, W_{t}$ is 3 -connected and $\left\{K_{4}, K_{2, m_{2}}\right\}$-free. Hence there exists $t \geqslant 6$ such that $W_{t}$ contains $T$ as an induced subgraph. Since $T \in \mathcal{T}_{0}$ and every member of $\mathcal{T}_{0}$ contained in $W_{t}$ as an induced subgraph is a path, $T$ is a path. Consequently (ii) of Theorem 1.1 holds.

Subcase 1.3: $m_{1}=2$ and $n=3$

Recall that $T \in \mathcal{T}_{0}$. Also if $3 \leqslant m_{2} \leqslant 4$, then $T \in \mathcal{T}_{1}$ by Lemma 2.4(i); if $m_{2} \geqslant 5$, then $T \in \mathcal{T}_{2}$ by Lemma 2.4(ii). Thus (iii) of Theorem 1.1 holds.

Case 2: $\mathcal{F}$ contains a star

Interchanging the roles of $K_{m_{1}, m_{2}}$ and $T$ with each other if necessary, we may assume that $K_{m_{1}, m_{2}}$ is the star of the smallest order contained in $\mathcal{F}$. Then $m_{1}=1$, and $m_{2} \geqslant 3$ by the assumption that $K_{1,2} \notin \mathcal{F}$, and $T \neq K_{1,2}, K_{1,3}$ by the minimality of $m_{2}$.

Subcase 2.1: $m_{2} \geqslant 4$

By Lemma 2.3, $T \in \mathcal{T}_{0}$.

Subcase 2.1.1: $n \geqslant 4$ 
For each $s \geqslant 7$, let $C_{s}^{2}$ denote the square of $C_{s}$; that is to say $V\left(C_{s}^{2}\right)=\left\{x_{i} \mid 1 \leqslant i \leqslant s\right\}$ and $E\left(C_{s}^{2}\right)=\left\{x_{i} x_{i+1}, x_{i} x_{i+2} \mid 1 \leqslant i \leqslant s\right\}$, where indices of the letter $x$ are to be read modulo $s$. Then $C_{s}^{2}$ is 3 -connected and $\left\{K_{n}, K_{1, m_{2}}\right\}$-free. Hence there exists $s \geqslant 7$ such that $C_{s}^{2}$ contains $T$ as an induced subgraph. Since every tree contained in $C_{s}^{2}$ as an induced subgraph is a path, $T$ is a path. Hence (iv) of Theorem 1.1 holds.

Subcase 2.1.2: $n=3$

If $m_{2}=4$, then $T \in \mathcal{T}_{1}$ by Lemma 2.4(i); if $m_{2} \geqslant 5$, then $T \in \mathcal{T}_{2}$ by Lemma 2.4(ii). Thus (v) of Theorem 1.1 holds.

Subcase 2.2: $m_{2}=3$

By the assumption that $\left\{K_{1,3}, K_{3}\right\} \nsubseteq \mathcal{F}$, we have $n \geqslant 4$ and $T \neq K_{3}$. Thus $T \in$ $\mathcal{G}-\left\{K_{1,2}, K_{1,3}, K_{3}\right\}$.

For a 3-connected 3-regular graph $G$, let $H_{G}$ be the graph obtained by expanding each vertex of $G$ to a triangle (see Figure 2); more precisely, we define $H_{G}$ by $V\left(H_{G}\right)=\left\{x_{u, v} \mid\right.$ $(u, v) \in V(G) \times V(G), u v \in E(G)\}$ and $E\left(H_{G}\right)=\left\{x_{u, v} x_{u, w} \mid u \in V(G), v, w \in N(u), v \neq\right.$ $w\} \cup\left\{x_{u, v} x_{v, u} \mid u v \in E(G)\right\}$. Then $H_{G}$ is 3-connected 3-regular and $\left\{K_{n}, K_{1,3}\right\}$-free and, for $u \in V(G), B_{u}=x_{u, v_{1}} x_{u, v_{2}} x_{u, v_{3}} x_{u, v_{1}}\left(\left\{v_{1}, v_{2}, v_{3}\right\}=N(u)\right)$ is a triangle of $H_{G}$. Also each cycle of $H_{G}$ which is not of the form $B_{u}$ with $u \in V(G)$ has length at least twice as large as the girth of $G$ and, for any $u, u^{\prime} \in V(G)$ with $u \neq u^{\prime}$, every induced path in $H_{G}$ joining $B_{u}$ and $B_{u^{\prime}}$ has even order. Hence every induced connected subgraph of $H_{G}$ having order greater than or equal to four and strictly less than twice the girth of $G$ belongs to $\mathcal{T}_{0}^{*}$. On the other hand, by Lemma 2.1, the set $\left\{H_{G} \mid G\right.$ is a 3-connected 3 -regular graph with girth at least $(|V(T)|+1) / 2\}$ is an infinite set. Hence there exists a 3-connected 3-regular graph $G$ with girth at least $(|V(T)|+1) / 2$ such that $H_{G}$ contains $T$ as an induced subgraph. Note that $|V(T)|$ is less than twice the girth of $G$. Consequently $T \in \mathcal{T}_{0}^{*}$.

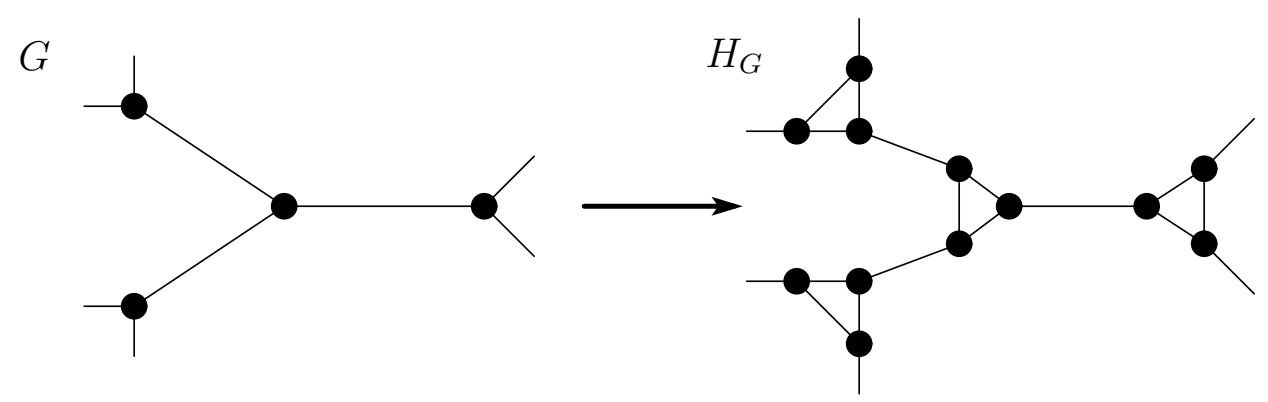

Figure 2: Graph $H_{G}$

Further for each $s \geqslant 7, C_{s}^{2}$ is 3 -connected and $\left\{K_{n}, K_{1,3}\right\}$-free. Hence there exists $s \geqslant 7$ such that $C_{s}^{2}$ contains $T$ as an induced subgraph. Since every member of $\mathcal{T}_{0}^{*}$ contained in $C_{s}^{2}$ as an induced subgraph belongs to $\mathcal{T}_{1}^{*}, T \in \mathcal{T}_{1}^{*}$.

Now assume $n \geqslant 5$. For each $s \geqslant 4$, let $C_{s}^{*}$ denote the lexicographic product of $C_{s}$ and $K_{2}$; that is to say, $V\left(C_{s}^{*}\right)=\left\{x_{i, j} \mid 1 \leqslant i \leqslant s, 1 \leqslant j \leqslant 2\right\}$ and $E\left(C_{s}^{*}\right)=\left\{x_{i, j} x_{i+1, h}, x_{i, 1} x_{i, 2} \mid\right.$ $1 \leqslant i \leqslant s, 1 \leqslant j, h \leqslant 2\}$, where first indices of the letter $x$ are to be read modulo $s$. Then 
$C_{s}^{*}$ is 3 -connected and $\left\{K_{n}, K_{1,3}\right\}$-free. Hence there exists $s \geqslant 4$ such that $C_{s}^{*}$ contains $T$ as an induced subgraph. Since every member of $\mathcal{T}_{1}^{*}$ contained in $C_{s}^{*}$ as an induced subgraph belongs to $\mathcal{T}_{2}^{*}, T \in \mathcal{T}_{2}^{*}$.

Thus we have $T \in \mathcal{T}_{1}^{*}$ if $n=4$, and $T \in \mathcal{T}_{2}^{*}$ if $n \geqslant 5$. Hence (vi) of Theorem 1.1 holds. This completes the proof of Theorem 1.1.

\section{$3 \quad K_{3, m}$-free graphs}

In this section, we prove Theorem 1.2. We first show that both $\mathcal{G}_{3}\left(\left\{K_{3}, K_{3, m}, Y_{3}\right\}\right)$ and $\mathcal{G}_{3}\left(\left\{K_{3}, K_{3, m}, P_{5}\right\}\right)$ are finite.

Proposition 3.1. Let $m \geqslant 4$. Then $\mathcal{G}_{3}\left(\left\{K_{3}, K_{3, m}, Y_{3}\right\}\right)$ is finite.

Proof. Let $G \in \mathcal{G}_{3}\left(\left\{K_{3}, K_{3, m}, Y_{3}\right\}\right)$. We show that $|V(G)| \leqslant(m+1)^{3}$.

Claim 3.1. $\operatorname{diam}(G) \leqslant 3$.

Proof. Suppose that $\operatorname{diam}(G) \geqslant 4$. Let $x, y \in V(G)$ be vertices with $d(x, y)=4$, and let $x_{0} x_{1} \cdots x_{4}$ be a shortest $x-y$ path in $G$. Since $G$ is 3 -connected, $N\left(x_{2}\right)-\left\{x_{1}, x_{3}\right\} \neq \varnothing$. Let $z \in N\left(x_{2}\right)-\left\{x_{1}, x_{3}\right\}$. Since $G$ is $K_{3}$-free, $z x_{1}, z x_{3} \notin E(G)$. Since $d\left(x_{0}, x_{4}\right)=4, z$ is adjacent to at most one of $x_{0}$ and $x_{4}$. Hence $\left\{x_{0}, x_{1}, x_{2}, x_{3}, z\right\}$ or $\left\{x_{4}, x_{3}, x_{2}, x_{1}, z\right\}$ induces $Y_{3}$, which is a contradiction.

In view of Claim 3.1 and Lemma 1.6, it suffices to show that $\Delta(G) \leqslant m+1$. Suppose that $\Delta(G) \geqslant m+2$. Let $w \in V(G)$ be a vertex such that $d(w)=\Delta(G)$, and let $x \in N(w)$. Since $G$ is $K_{3}$-free, both $N(w)$ and $N(x)$ are independent. Since $G$ is 3-connected, $d(x) \geqslant$ 3. Take $y_{1}, y_{2} \in N(x)-\{w\}$. If $\left|N(w)-N\left(y_{i}\right)\right| \geqslant 2$ for $i=1$ or 2 , say $a, b \in N(w)-N\left(y_{i}\right)$, then $\left\{y_{i}, x, w, a, b\right\}$ induces $Y_{3}$, a contradiction. Thus $\left|N\left(y_{i}\right) \cap N(w)\right| \geqslant d(w)-1$ for each $i=1,2$. Consequently $\left|N(w) \cap N\left(y_{1}\right) \cap N\left(y_{2}\right)\right| \geqslant d(w)-2 \geqslant m$, which implies that $G\left[\left\{w, y_{1}, y_{2}\right\} \cup\left(N(w) \cap N\left(y_{1}\right) \cap N\left(y_{2}\right)\right)\right]$ contains $K_{3, m}$ as an induced subgraph, a contradiction.

Proposition 3.2. Let $m \geqslant 4$. Then $\mathcal{G}_{3}\left(\left\{K_{3}, K_{3, m}, P_{5}\right\}\right)$ is finite.

Proof. Let $G \in \mathcal{G}_{3}\left(\left\{K_{3}, K_{3, m}, P_{5}\right\}\right)$. We show that $|V(G)| \leqslant(4 m-1)^{3}$. Since $G$ is $P_{5^{-}}$ free, $\operatorname{diam}(G) \leqslant 3$. Thus in view of Lemma 1.6, it suffices to show that $\Delta(G) \leqslant 4 m-1$. Suppose that $\Delta(G) \geqslant 4 m$, and let $w \in V(G)$ be a vertex with $d(w)=\Delta(G)$. Since $G$ is $K_{3}$-free, $N(w)$ is an independent set.

Claim 3.2. Let $X \subseteq N(w)$, and let $Y$ be a minimal subset of $N_{2}(w)$ such that $N(Y) \supseteq X$. Then $|Y| \leqslant 2$.

Proof. Suppose that $|Y| \geqslant 3$. Since $G$ is $K_{3}$-free, there exist vertices $y_{1}, y_{2} \in Y$ such that $y_{1} y_{2} \notin E(G)$. By the minimality of $Y,\left(N\left(y_{i}\right) \cap X\right)-N\left(y_{3-i}\right) \neq \varnothing$ for each $i=1,2$. For $i=1,2$, let $x_{i} \in\left(N\left(y_{i}\right) \cap X\right)-N\left(y_{3-i}\right)$. Then $\left\{y_{1}, x_{1}, w, x_{2}, y_{2}\right\}$ induces $P_{5}$, a contradiction.

Since $G$ is 3-connected and $N(w)$ is independent, $N\left(N_{2}(w)\right) \supseteq N(w)$. Let $Y$ be a minimal subset of $N_{2}(w)$ such that $N(Y) \supseteq N(w)$. Then $|Y| \leqslant 2$ by Claim 3.2. 
Hence there exists $y \in Y$ such that $|N(w) \cap N(y)| \geqslant d(w) / 2 \geqslant 2 m$. Since $G$ is 3 connected and $N(w)$ is independent, $N\left(N_{2}(w)-\{y\}\right) \supseteq N(w) \cap N(y)$. Let $Y^{*}$ be a minimal subset of $N_{2}(w)-\{y\}$ such that $N\left(Y^{*}\right) \supseteq N(w) \cap N(y)$ (see Figure 3). Then $\left|Y^{*}\right| \leqslant 2$ by Claim 3.2. Hence there exists $y^{\prime} \in Y^{*}$ such that $\left|N(w) \cap N(y) \cap N\left(y^{\prime}\right)\right| \geqslant m$. Since $N(y) \cap N\left(y^{\prime}\right) \neq \varnothing$, we have $y y^{\prime} \notin E(G)$ by the assumption that $G$ is $K_{3}$-free. Consequently $G\left[\left\{w, y, y^{\prime}\right\} \cup\left(N(w) \cap N(y) \cap N\left(y^{\prime}\right)\right)\right]$ contains $K_{3, m}$ as an induced subgraph, a contradiction.

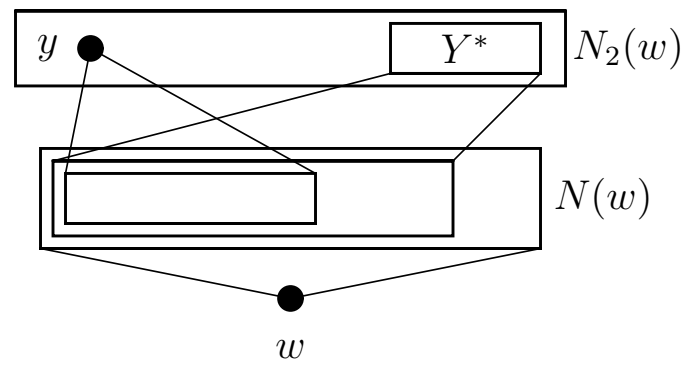

Figure 3: Vertex $y$ and set $Y^{*}$

Proof of Theorem 1.2. The 'if' part follows from Propositions 3.1 and 3.2. Thus it suffices to prove the 'only if' part. Suppose that $\mathcal{G}_{3}\left(\left\{K_{3}, K_{3, m}, T\right\}\right)$ is finite. From Theorem 1.1, it follows that $T \in \mathcal{T}_{2}$. For each $s \geqslant 2$, let $H_{s}$ be the graph defined by $V\left(H_{s}\right)=\left\{x_{i, j} \mid\right.$ $1 \leqslant i, j \leqslant 2\} \cup\left\{y_{i, j} \mid 1 \leqslant i \leqslant 2,1 \leqslant j \leqslant s\right\}$ and $E\left(H_{s}\right)=\left\{x_{1, j} x_{2, h} \mid 1 \leqslant j, h \leqslant\right.$ $2\} \cup\left\{x_{i, j} y_{i, h} \mid 1 \leqslant i, j \leqslant 2,1 \leqslant h \leqslant s\right\} \cup\left\{y_{1, j} y_{2, j} \mid 1 \leqslant j \leqslant s\right\}$. Then $H_{s}$ is 3 -connected and $\left\{K_{3}, K_{3, m}\right\}$-free since $m \geqslant 4$. Since $\mathcal{G}_{3}\left(\left\{K_{3}, K_{3, m}, T\right\}\right)$ is finite, there exists $s \geqslant 2$ such that $H_{s}$ contains $T$ as an induced subgraph. By inspection, we see that if $F$ is a member of $\mathcal{T}_{2}$ contained in $H_{s}$ as an induced subgraph, then $F$ is a path of order 4 or 5 or $F=Y_{3}$. Hence $T$ is a path of order 4 or 5 or $T=Y_{3}$.

\section{$4\left\{K_{4}, K_{2, m}\right\}$-free graphs}

In this section, we prove Theorem 1.3.

Proposition 4.1. Let $m \geqslant 3$. Then $\mathcal{G}_{3}\left(\left\{K_{4}, K_{2, m}, P_{5}\right\}\right)$ is finite.

Proof. By part (i) of Theorem B, there exists a positive integer $t=t(m)$ such that every 2connected $\left\{K_{3}, K_{2, m}, P_{5}\right\}$-free graph has order at most $t$. Let $G \in \mathcal{G}_{3}\left(\left\{K_{4}, K_{2, m}, P_{5}\right\}\right)$. We show that $|V(G)| \leqslant(3(3 m-1) t / 2)^{3}$. Note that $\operatorname{diam}(G) \leqslant 3$. Thus in view of Lemma 1.6, it suffices to show that $\Delta(G) \leqslant 3(3 m-1) t / 2$. Suppose that $\Delta(G)>3(3 m-1) t / 2$, and let $w \in V(G)$ be a vertex with $d(w)=\Delta(G)$. Since $G$ is $\left\{K_{4}, K_{2, m}, P_{5}\right\}$-free, $G[N(w)]$ is $\left\{K_{3}, K_{2, m}, P_{5}\right\}$-free.

Let $F$ be a component of $G[N(w)]$. If $F$ has two or more blocks which are not endblocks, then $F$ contains $P_{5}$ as an induced subgraph, a contradiction. Thus $F$ has at most one block which is not an endblock, which implies that at least two thirds of the blocks of $F$ are endblocks. Since $F$ is arbitrary, it follows that at least two thirds of the blocks of $G[N(w)]$ are endblocks. Now suppose that $G[N(w)]$ has at most $3 m-1$ 
endblocks. Then $G[N(w)]$ has at most $3(3 m-1) / 2$ blocks. Since $d(w)>3(3 m-1) t / 2$, it follows that there exists a block $B$ of $G[N(w)]$ with $|V(B)|>t$. Since $G[N(w)]$ is $\left\{K_{3}, K_{2, m}, P_{5}\right\}$-free, this contradicts the definition of $t$. Thus $G[N(w)]$ has at least $3 m$ endblocks. Let $B_{1}, \ldots, B_{3 m}$ be endblocks of $G[N(w)]$. Since $G$ is 3-connected, we see that for each $1 \leqslant i \leqslant 3 m$, there exists an internal vertex $x_{i}$ of $B_{i}$ such that $N\left(x_{i}\right) \cap(V(G)-$ $(\{w\} \cup N(w))) \neq \varnothing$ (see Figure 4). Set $X=\left\{x_{1}, \ldots, x_{3 m}\right\}$. Then $X$ is an independent set of $G$, and we have $N\left(N_{2}(w)\right) \supseteq X$. Let $Y$ be a minimal subset of $N_{2}(w)$ such that $N(Y) \supseteq X$. If $|Y| \leqslant 3$, then there exists $y \in Y$ such that $|N(y) \cap X| \geqslant m$, and hence $G[\{w, y\} \cup(N(y) \cap X)]$ contains $K_{2, m}$ as an induced subgraph, a contradiction. Thus $|Y| \geqslant 4$. Since $G$ is $K_{4}$-free, there exist vertices $y_{1}, y_{2} \in Y$ such that $y_{1} y_{2} \notin E(G)$. By the minimality of $Y,\left(N\left(y_{i}\right) \cap X\right)-N\left(y_{3-i}\right) \neq \varnothing$ for each $i=1,2$. For $i=1,2$, let $x_{i}^{\prime} \in\left(N\left(y_{i}\right) \cap X\right)-N\left(y_{3-i}\right)$. Then $\left\{y_{1}, x_{1}^{\prime}, w, x_{2}^{\prime}, y_{2}\right\}$ induces $P_{5}$, a contradiction.

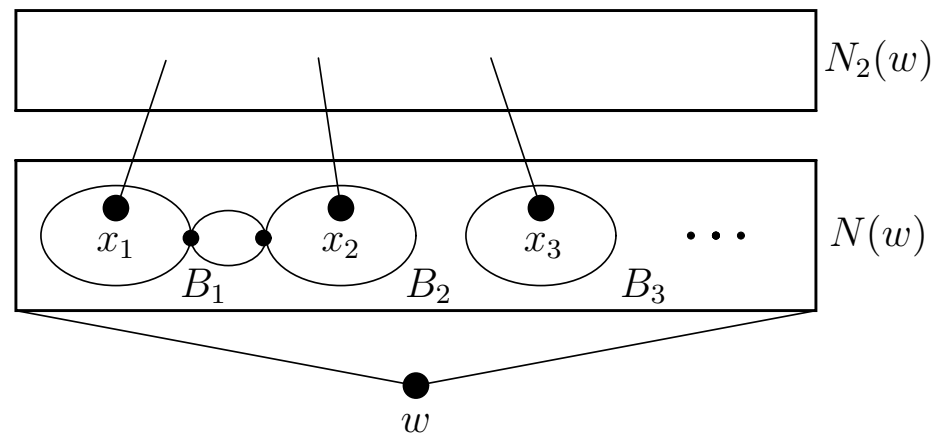

Figure 4: Endblocks $B_{i}$ and vertices $x_{i}$

Proof of Theorem 1.3. The 'if' part follows from Proposition 4.1. Thus it suffices to prove the 'only if' part. Suppose that $\mathcal{G}_{3}\left(\left\{K_{4}, K_{2, m}, T\right\}\right)$ is finite. From Theorem 1.1, it follows that $T$ is a path. For each $s \geqslant 2$, let $H_{s}$ be the graph defined by $V\left(H_{s}\right)=\left\{x_{i}, y_{i, j} \mid 1 \leqslant i \leqslant\right.$ $3,1 \leqslant j \leqslant s\}$ and $E\left(H_{s}\right)=\left\{x_{i} x_{j}, y_{i, h} y_{j, h} \mid i \neq j, 1 \leqslant h \leqslant s\right\} \cup\left\{x_{i} y_{j, h} \mid i \neq j, 1 \leqslant h \leqslant s\right\}$ (see Figure 5). Then $H_{s}$ is 3 -connected and $\left\{K_{4}, K_{2, m}\right\}$-free. Hence there exists $s \geqslant 2$ such that $H_{s}$ contains $T$ as an induced subgraph. Since every induced path of $H_{s}$ has order at most $5, T$ has order at most 5 . Since $T \neq K_{1,2}$ by assumption, it follows that $T$ is a path of order 4 or 5 .

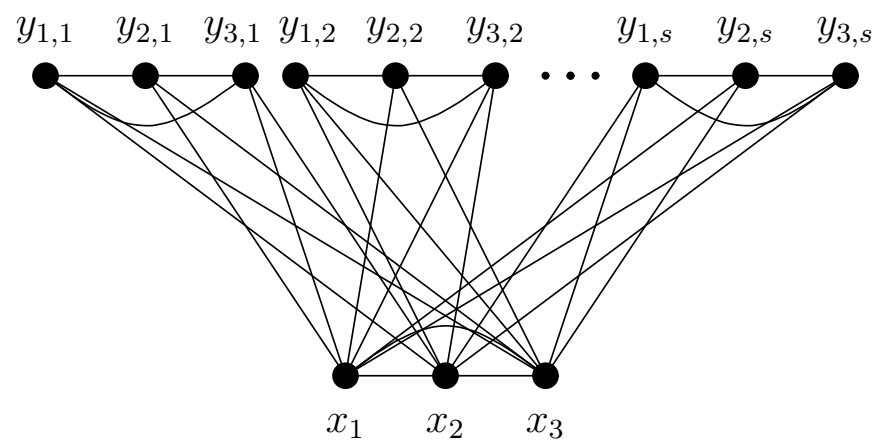

Figure 5: Graph $H_{s}$ 


\section{$5 \quad K_{1, m}$-free graphs}

In this section, we prove Theorem 1.5. We first prove a lemma, which we also use in Section 6 .

Lemma 5.1. Let $n \geqslant 3$, and let $G$ be a 3-connected $\left\{K_{3}, Y_{n}^{*}\right\}$-free graph. If $n=3$, let $t(n)=7$; if $n \geqslant 4$, let $t(n)=3 n+10$. Then $\operatorname{diam}(G) \leqslant t(n)$. In particular, $\operatorname{diam}(G) \leqslant 3 n+10$.

Proof. Let $t=t(n)$, and suppose that $\operatorname{diam}(G) \geqslant t+1$. Let $x, y \in V(G)$ be vertices such that $d(x, y)=t+1$, and let $P=x_{0} x_{1} \cdots x_{t+1}$ be a shortest $x-y$ path in $G$. Since $G$ is 3-connected, $N\left(x_{i}\right)-\left\{x_{i-1}, x_{i+1}\right\} \neq \varnothing$ for each $1 \leqslant i \leqslant t$. Let $x_{i}^{\prime} \in N\left(x_{i}\right)-\left\{x_{i-1}, x_{i+1}\right\}$.

Case 1: $n=3$

We first show that $N\left(x_{3}\right) \cap N\left(x_{5}\right)=\left\{x_{4}\right\}$. By way of contradiction, suppose that $\left|N\left(x_{3}\right) \cap N\left(x_{5}\right)\right| \geqslant 2$. Then we may assume we have chosen $x_{3}^{\prime}$ and $x_{5}^{\prime}$ so that $x_{3}^{\prime}=x_{5}^{\prime} \in$ $\left(N\left(x_{3}\right) \cap N\left(x_{5}\right)\right)-\left\{x_{4}\right\}$. Since $d\left(x_{0}, x_{8}\right)=8$ and $G$ is $K_{3}$-free, we get $N\left(x_{3}^{\prime}\right) \cap V(P)=$ $\left\{x_{3}, x_{5}\right\}, N\left(x_{1}^{\prime}\right) \cap V(P) \subseteq\left\{x_{1}, x_{3}\right\}, N\left(x_{7}^{\prime}\right) \cap V(P) \subseteq\left\{x_{5}, x_{7}\right\}$, and $x_{1}^{\prime} x_{3}^{\prime}, x_{3}^{\prime} x_{7}^{\prime} \notin E(G)$. Since neither $\left\{x_{0}, x_{1}^{\prime}, x_{1}, x_{2}, x_{3}, x_{4}, x_{3}^{\prime}\right\}$ nor $\left\{x_{4}, x_{3}^{\prime}, x_{5}, x_{6}, x_{7}, x_{8}, x_{7}^{\prime}\right\}$ induces $Y_{3}^{*}$, this forces $N\left(x_{1}^{\prime}\right) \cap V(P)=\left\{x_{1}, x_{3}\right\}$ and $N\left(x_{7}^{\prime}\right) \cap V(P)=\left\{x_{5}, x_{7}\right\}$. But then $\left\{x_{2}, x_{1}^{\prime}, x_{3}, x_{4}, x_{5}, x_{6}, x_{7}^{\prime}\right\}$ induces $Y_{3}^{*}$, a contradiction. Thus $N\left(x_{3}\right) \cap N\left(x_{5}\right)=\left\{x_{4}\right\}$.

Choose $z \in N\left(x_{3}\right)-\left\{x_{2}, x_{4}\right\}$. Since $\left\{x_{2}, z, x_{3}, x_{4}, x_{5}, x_{6}, x_{5}^{\prime}\right\}$ does not induce $Y_{3}^{*}$ and $N\left(x_{3}\right) \cap N\left(x_{5}\right)=\left\{x_{4}\right\}$, we get $z x_{5}^{\prime} \in E(G)$. Since $d\left(x_{1}, x_{5}\right)=4$, it follows that $x_{1} z \notin E(G)$. Since $z \in N\left(x_{3}\right)-\left\{x_{2}, x_{4}\right\}$ is arbitrary, this implies $N\left(x_{1}\right) \cap N\left(x_{3}\right)=\left\{x_{2}\right\}$; in particular, $x_{1}^{\prime} x_{3} \notin E(G)$. Letting $z=x_{3}^{\prime}$, we also get $x_{3}^{\prime} x_{5}^{\prime} \in E(G)$ and $x_{1} x_{3}^{\prime} \notin E(G)$. Since $\left\{x_{0}, x_{1}^{\prime}, x_{1}, x_{2}, x_{3}, x_{4}, x_{3}^{\prime}\right\}$ does not induce $Y_{3}^{*}$, we now obtain $x_{1}^{\prime} x_{3}^{\prime} \in E(G)$. Similarly, $x_{7}^{\prime} x_{5}^{\prime} \in E(G)$. But then $x_{0} x_{1} x_{1}^{\prime} x_{3}^{\prime} x_{5}^{\prime} x_{7}^{\prime} x_{7} x_{8}$ is an $x_{0}-x_{8}$ path of length 7 , which contradicts the fact that $d\left(x_{0}, x_{8}\right)=8$.

Case 2: $n \geqslant 4$

Claim 5.1. For $0 \leqslant i \leqslant t-n-1$, if $\left|N\left(x_{i}\right) \cap N\left(x_{i+2}\right)\right| \geqslant 2$, then $x_{i+n+1}^{\prime} x_{i+n-1} \in E(G)$. For $n \leqslant i \leqslant t-1$, if $\left|N\left(x_{i}\right) \cap N\left(x_{i+2}\right)\right| \geqslant 2$, then $x_{i-n+1}^{\prime} x_{i-n+3} \in E(G)$.

Proof. Let $0 \leqslant i \leqslant t-n-1$, and assume that $\left|N\left(x_{i}\right) \cap N\left(x_{i+2}\right)\right| \geqslant 2$. Let $u \in\left(N\left(x_{i}\right) \cap\right.$ $\left.N\left(x_{i+2}\right)\right)-\left\{x_{i+1}\right\}$. Since $d\left(x_{0}, x_{t+1}\right)=t+1$ and $G$ is $K_{3}$-free, we have $N(u) \cap V(P)=$ $\left\{x_{i}, x_{i+2}\right\}, N\left(x_{i+n+1}^{\prime}\right) \cap\left\{x_{0}, x_{1}, \ldots, x_{i+n+2}\right\} \subseteq\left\{x_{i+n-1}, x_{i+n+1}\right\}$ and $u x_{i+n+1}^{\prime} \notin E(G)$. Since $\left\{x_{i+1}, u, x_{i+2}, \ldots, x_{i+n+1}, x_{i+n+2}, x_{i+n+1}^{\prime}\right\}$ does not induce $Y_{n}^{*}$, this implies $x_{i+n+1}^{\prime} x_{i+n-1} \in$ $E(G)$. Thus the first assertion is proved. We can similarly verify the second assertion.

Claim 5.2. There is no integer $i$ with $n \leqslant i \leqslant t-n-2$ such that $\left|N\left(x_{i}\right) \cap N\left(x_{i+2}\right)\right| \geqslant 2$ and $\left|N\left(x_{i+1}\right) \cap N\left(x_{i+3}\right)\right| \geqslant 2$.

Proof. Suppose that there exists an integer $i$ with $n \leqslant i \leqslant t-n-2$ such that $\mid N\left(x_{i}\right) \cap$ $N\left(x_{i+2}\right) \mid \geqslant 2$ and $\left|N\left(x_{i+1}\right) \cap N\left(x_{i+3}\right)\right| \geqslant 2$. Then by Claim 5.1, $x_{i-n+1}^{\prime} x_{i-n+3}, x_{i+n+2}^{\prime} x_{i+n} \in$ $E(G)$. This implies $\left|N\left(x_{i-n+1}\right) \cap N\left(x_{i-n+3}\right)\right| \geqslant 2$ and $\left|N\left(x_{i+n}\right) \cap N\left(x_{i+n+2}\right)\right| \geqslant 2$. Let $\mathcal{Q}$ be the set of $x_{i}-x_{i+3}$ paths of order 4 , and let $X=\left(\bigcup_{Q \in \mathcal{Q}} V(Q)\right)-\left\{x_{i}, x_{i+3}\right\}$. Since 
$G-\left\{x_{i}, x_{i+3}\right\}$ is connected and $V(G)-\left(X \cup\left\{x_{i}, x_{i+3}\right\}\right) \neq \varnothing$, there exists $z \in X$ such that $N(z)-\left(X \cup\left\{x_{i}, x_{i+3}\right\}\right) \neq \varnothing$. By the definition of $X, z$ is adjacent to $x_{i}$ or $x_{i+3}$. By symmetry, we may assume $z x_{i} \in E(G)$. Then there exists $z^{\prime} \in X$ such that $x_{i} z z^{\prime} x_{i+3}$ is an $x_{i}-x_{i+3}$ path. Now take $w \in N(z)-\left(X \cup\left\{x_{i}, x_{i+3}\right\}\right)$. Since $\left|N\left(x_{i+n}\right) \cap N\left(x_{i+n+2}\right)\right| \geqslant 2$, we obtain $w x_{i+3} \in E(G)$ by applying the second assertion of Claim 5.1 to the path $x_{0} x_{1} \cdots x_{i} z z^{\prime} x_{i+3} x_{i+4} \cdots x_{t+1}$. But then $x_{i} z w x_{i+3} \in \mathcal{Q}$, which contradicts the fact that $w \notin X$.

Claim 5.3. For $n+2 \leqslant i \leqslant t-2 n$, we have $x_{i-1}^{\prime} x_{i+n-2}^{\prime} \in E(G)$ or $x_{i}^{\prime} x_{i+n-1}^{\prime} \in E(G)$ or $x_{i+1}^{\prime} x_{i+n}^{\prime} \in E(G)$.

Proof. Suppose that $x_{i}^{\prime} x_{i+n-1}^{\prime} \notin E(G)$. Since $\left\{x_{i-1}, x_{i}^{\prime}, x_{i}, \ldots, x_{i+n-1}, x_{i+n}, x_{i+n-1}^{\prime}\right\}$ does not induce $Y_{n}^{*}, x_{i}^{\prime} x_{i+2} \in E(G)$ or $x_{i+n-1}^{\prime} x_{i+n-3} \in E(G)$. First assume that $x_{i}^{\prime} x_{i+2} \in E(G)$. Since $0 \leqslant i \leqslant t-n-1$, we have $x_{i+n+1}^{\prime} x_{i+n-1} \in E(G)$ by Claim 5.1. Since $n \leqslant i \leqslant t-n-2$ and $n \leqslant i+n-2 \leqslant t-n-2, x_{i+1}^{\prime} x_{i+3}, x_{i+n}^{\prime} x_{i+n-2} \notin E(G)$ by Claim 5.2. Since $\left\{x_{i}, x_{i+1}^{\prime}, x_{i+1}, \ldots, x_{i+n}, x_{i+n+1}, x_{i+n}^{\prime}\right\}$ does not induce $Y_{n}^{*}$ and $G$ is $K_{3}$-free, it follows that $x_{i+1}^{\prime} x_{i+n}^{\prime} \in E(G)$, as desired. Next assume that $x_{i+n-1}^{\prime} x_{i+n-3} \in E(G)$. Arguing as above, we get $x_{i-2}^{\prime} x_{i} \in E(G)$ by Claim 5.1, and $x_{i-1}^{\prime} x_{i+1}, x_{i+n-2}^{\prime} x_{i+n-4} \notin E(G)$ by Claim 5.2. Since $\left\{x_{i-2}, x_{i-1}^{\prime}, x_{i-1}, \ldots, x_{i+n-2}, x_{i+n-1}, x_{i+n-2}^{\prime}\right\}$ does not induce $Y_{n}^{*}$ and $G$ is $K_{3}$-free, it follows that $x_{i-1}^{\prime} x_{i+n-2}^{\prime} \in E(G)$, as desired.

Let $j=n+2$. Since $t \geqslant 3 n+10$, we have $n+2 \leqslant j \leqslant t-2 n$. Hence it follows from Claim 5.3 that $x_{j-1}^{\prime} x_{j+n-2}^{\prime} \in E(G)$ or $x_{j}^{\prime} x_{j+n-1}^{\prime} \in E(G)$ or $x_{j+1}^{\prime} x_{j+n}^{\prime} \in E(G)$. Since $d\left(x_{j-1}, x_{j+n-2}\right)=d\left(x_{j}, x_{j+n-1}\right)=d\left(x_{j+1}, x_{j+n}\right)=n-1$, this forces $n=4$, and hence $t=22$ and $j=6$. In particular, $x_{5}^{\prime} x_{8}^{\prime} \in E(G)$ or $x_{6}^{\prime} x_{9}^{\prime} \in E(G)$ or $x_{7}^{\prime} x_{10}^{\prime} \in E(G)$. Let $s \in\{5,6,7\}$ be an integer such that $x_{s}^{\prime} x_{s+3}^{\prime} \in E(G)$.

Claim 5.4. For $4 \leqslant i \leqslant 11$, if $x_{i}^{\prime} x_{i+3}^{\prime} \in E(G)$, then $x_{i+2}^{\prime} x_{i+5}^{\prime} \in E(G)$.

Proof. If $x_{i+3}^{\prime} x_{i+6}^{\prime} \in E(G)$, then $x_{i} x_{i}^{\prime} x_{i+3}^{\prime} x_{i+6}^{\prime} x_{i+6}$ is a path, which contradicts the fact that $d\left(x_{i}, x_{i+6}\right)=6$. Thus $x_{i+3}^{\prime} x_{i+6}^{\prime} \notin E(G)$. Suppose that $x_{i+2}^{\prime} x_{i+5}^{\prime} \notin E(G)$. Then $x_{i+2}^{\prime} x_{i+5}^{\prime}, x_{i+3}^{\prime} x_{i+6}^{\prime} \notin E(G)$. Since $n+2=6 \leqslant i+2<i+3 \leqslant 14=t-2 n$, this together with Claim 5.3 implies $x_{i+1}^{\prime} x_{i+4}^{\prime} \in E(G)$ and $x_{i+4}^{\prime} x_{i+7}^{\prime} \in E(G)$. It now follows that $x_{i+1} x_{i+1}^{\prime} x_{i+4}^{\prime} x_{i+7}^{\prime} x_{i+7}$ is a path, which contradicts the fact that $d\left(x_{i+1}, x_{i+7}\right)=6$.

Recall that $x_{s}^{\prime} x_{s+3}^{\prime} \in E(G)$. Since $4<s<s+2<s+4 \leqslant 11$, by repetitively applying Claim 5.4, we obtain $x_{s+2}^{\prime} x_{s+5}^{\prime}, x_{s+4}^{\prime} x_{s+7}^{\prime}, x_{s+6}^{\prime} x_{s+9}^{\prime} \in E(G)$. Since $x_{s}^{\prime} x_{s+3}^{\prime}, x_{s+6}^{\prime} x_{s+9}^{\prime} \in$ $E(G)$ and $d\left(x_{s}, x_{s+9}\right)=9$, we get $N\left(x_{s+3}^{\prime}\right) \cap\left\{x_{s+5}, x_{s+6}, x_{s+7}, x_{s+6}^{\prime}\right\}=\varnothing$ and $N\left(x_{s+6}^{\prime}\right) \cap$ $\left\{x_{s+2}, x_{s+3}^{\prime}, x_{s+3}, x_{s+4}\right\}=\varnothing$. Since $G$ is $K_{3}$-free, it follows that $\left\{x_{s+2}, x_{s+3}^{\prime}, x_{s+3}, x_{s+4}, x_{s+5}\right.$, $\left.x_{s+6}, x_{s+7}, x_{s+6}^{\prime}\right\}$ induces $Y_{4}^{*}$, which is a contradiction.

This completes the proof of Lemma 5.1.

Proof of Theorem 1.5. Let $n \geqslant 3$, and let $G \in \mathcal{G}_{3}\left(\left\{K_{3}, K_{1, m}, Y_{n}^{*}\right\}\right)$. Then by Lemma 5.1, $\operatorname{diam}(G) \leqslant 3 n+10$. Since $G$ is $\left\{K_{3}, K_{1, m}\right\}$-free, we also have $\Delta(G) \leqslant m-1$. Hence $|V(G)| \leqslant(m-1)^{3 n+10}$ by Lemma 1.6. Thus $\mathcal{G}_{3}\left(\left\{K_{3}, K_{1, m}, Y_{n}^{*}\right\}\right)$ is finite. Since $n \geqslant 3$ is arbitrary, this proves the 'if' part. The 'only if' part follows from Theorem 1.1. 


\section{$6\left\{K_{3}, K_{2, m}\right\}$-free graphs}

In this section, we prove Theorem 1.4. We first prove several lemmas.

Lemma 6.1. Let $m \geqslant 2$, and let $G$ be a $\left\{K_{3}, K_{2, m}\right\}$-free graph. Let $H$ be a connected induced subgraph of $G$ with order $n \geqslant 2$ and let $x \in V(H)$, and suppose that $d_{G}(x) \geqslant$ $(m-1)(n-2)+t+1$. Then $G$ contains as an induced subgraph the graph obtained from $H$ by adding $t$ pendant edges to $x$.

Proof. Since $G$ is $K_{3}$-free, $N_{G}(x)-V(H)$ is independent and no vertex in $N_{G}(x)-V(H)$ is adjacent to a vertex in $N_{G}(x) \cap V(H)$. Since $G$ is $K_{2, m}$-free, we see that for each $x^{\prime} \in V(H)-\left(\{x\} \cup N_{G}(x)\right), x^{\prime}$ is adjacent to at most $m-1$ vertices in $N_{G}(x)-V(H)$. Set $Y=N_{G}(x) \cap\left(\left(\bigcup_{x^{\prime} \in V(H)-\{x\}} N_{G}\left(x^{\prime}\right)\right) \cup V(H)\right)$. It follows that $|Y| \leqslant(m-1)(n-1-$ $\left.d_{H}(x)\right)+d_{H}(x) \leqslant(m-1)(n-2)+1$. Hence $\left|N_{G}(x)-Y\right| \geqslant t$. Now if we let $Z$ be a subset of $N_{G}(x)-Y$ with $|Z|=t$, then $V(H) \cup Z$ induces the desired graph.

Lemma 6.2. Let $m \geqslant 2$, and let $G$ be a $\left\{K_{3}, K_{2, m}\right\}$-free graph. Let $P$ be an induced path of $G$ with order $n \geqslant 2$, and suppose that both endvertices of $P$ have degree at least $(m-1)(n-1)+2$. Then $G$ contains $P_{n+2}$ as an induced subgraph.

Proof. Applying Lemma 6.1 to one of the endvertices of $P$ with $H=P$, we get an induced path $P^{\prime}$ of order $n+1$ and, applying Lemma 6.1 to the other endvertices of $P$ with $H=P^{\prime}$, we obtain a path of the type desired.

Similarly, we obtain the following lemma.

Lemma 6.3. Let $m \geqslant 2$, and let $G$ be a $\left\{K_{3}, K_{2, m}\right\}$-free graph, and let $P$ be an induced path of $G$ with order $n \geqslant 2$, and suppose that both endvertices of $P$ have degree at least $(m-1) n+3$. Then $G$ contains $Y_{n}^{*}$ as an induced subgraph.

Proposition 6.4. Let $m \geqslant 5$. Then $\mathcal{G}_{3}\left(\left\{K_{3}, K_{2, m}, Y_{3}^{*}\right\}\right)$ is finite.

Proof. Let $G \in \mathcal{G}_{3}\left(\left\{K_{3}, K_{2, m}, Y_{3}^{*}\right\}\right)$. We show that $|V(G)| \leqslant((6 m-3)(3 m-2)(m-2)+1)^{7}$. By Lemma 5.1, $\operatorname{diam}(G) \leqslant 7$. Thus it suffices to show that $\Delta(G) \leqslant(6 m-3)(3 m-2)(m-$ $2)+1$. Suppose that $\Delta(G) \geqslant(6 m-3)(3 m-2)(m-2)+2$, and let $w \in V(G)$ be a vertex with $d(w)=\Delta(G)$. Since $G$ is $K_{3}$-free, $N(w)$ is independent. In particular, for any two vertices $x, x^{\prime}$ in $N(w),\left\{x, w, x^{\prime}\right\}$ induces $P_{3}$. Choose $a \in N(w)$ so that $d(a) \geqslant d(x)$ for all $x \in N(w)$. If two vertices in $N(w)$ have degree at least $3 m$, then by Lemma 6.3, $G$ contains $Y_{3}^{*}$ as an induced subgraph, a contradiction. Thus all vertex in $N(w)-\{a\}$ have degree at most $3 m-1$. Let $B \subseteq N(w)-\{a\}$ be a maximal set such that $N(b) \cap N\left(b^{\prime}\right)=\{w\}$ for any $b, b^{\prime} \in B$ with $b \neq b^{\prime}$.

Suppose that $|B| \leqslant 6 m-3$. Since $B \subseteq N(w)-\{a\},\left|\left(\bigcup_{b \in B} N(b)\right)-\{w\}\right| \leqslant(6 m-$ $3)(3 m-2)$. Since $G$ is $K_{2, m}$-free and $\left(\bigcup_{b \in B} N(b)\right)-\{w\} \subseteq N_{2}(w),|N(y) \cap N(w)| \leqslant m-1$ for all $y \in\left(\bigcup_{b \in B} N(b)\right)-\{w\}$. Hence $\left|\left\{x \in N(w) \mid N(x) \cap\left(\left(\bigcup_{b \in B} N(b)\right)-\{w\}\right) \neq \varnothing\right\}\right| \leqslant$ $(6 m-3)(3 m-2)(m-1)$. Since $|N(w)-\{a\}| \geqslant(6 m-3)(3 m-2)(m-1)+1$, there exists $b^{*} \in N(w)-\{a\}$ such that $\left(N\left(b^{*}\right) \cap\left(\bigcup_{b \in B} N(b)\right)\right)-\{w\}=\varnothing$. Then $B \cup\left\{b^{*}\right\}$ satisfies the condition that $N(b) \cap N\left(b^{\prime}\right)=\{w\}$ for any $b, b^{\prime} \in B \cup\left\{b^{*}\right\}$ with $b \neq b^{\prime}$, which contradicts the maximality of $B$. Thus $|B| \geqslant 6 m-2$. 
Since $G$ is 3-connected, $|N(b)-\{w\}| \geqslant 2$ for every $b \in B$. For each $b \in B$, let $v_{b}, u_{b} \in$ $N(b)-\{w\}$. Fix a vertex $b_{0} \in B$. For each $b \in B-\left\{b_{0}\right\}$, we have $E\left(\left\{v_{b_{0}}, u_{b_{0}}\right\},\left\{v_{b}, u_{b}\right\}\right) \neq \varnothing$ because $\left\{v_{b_{0}}, u_{b_{0}}, b_{0}, w, b, v_{b}, u_{b}\right\}$ does not induce $Y_{3}^{*}$. Since $\left|B-\left\{b_{0}\right\}\right| \geqslant 6 m-3$, this implies that $v_{b_{0}}$ or $u_{b_{0}}$ is adjacent to at least $3 m-1$ vertices in $\left\{v_{b}, u_{b} \mid b \in B-\left\{b_{0}\right\}\right\}$. We may assume $\left|N\left(v_{b_{0}}\right) \cap\left\{v_{b}, u_{b} \mid b \in B-\left\{b_{0}\right\}\right\}\right| \geqslant 3 m-1$. Then $d\left(v_{b_{0}}\right) \geqslant 3 m$. Since $d(w) \geqslant 3 m$ and $w b_{0} v_{b_{0}}$ is an induced path of $G$ of order $3, G$ contains $Y_{3}^{*}$ as an induced subgraph by Lemma 6.3, a contradiction.

This completes the proof of Proposition 6.4.

Before proving the finiteness of $\mathcal{G}_{3}\left(\left\{K_{3}, K_{2, m}, P_{7}\right\}\right)$, we state an important part of the proof as a separate lemma.

Lemma 6.5. Let $m \geqslant 5$, and let $G \in \mathcal{G}_{3}\left(\left\{K_{3}, K_{2, m}, P_{7}\right\}\right)$. Then there exist no vertices $a, b \in V(G)$ such that $d(a, b)=2, d(a) \geqslant 2(m-1)^{7}$ and $d(b) \geqslant 2(m-1)^{7}$.

Proof. Suppose that there exist two vertices $a, b \in V(G)$ such that $d(a, b)=2, d(a) \geqslant$ $2(m-1)^{7}$ and $d(b) \geqslant 2(m-1)^{7}$. Since $d(a, b)=2, a b \notin E(G)$. Let $w \in N(a) \cap N(b)$, and let $A=N(a)-N(b)$ and $B=N(b)-N(a)$. Since $G$ is $K_{3}$-free, $N(w), N(a)$ and $N(b)$ are independent sets, and hence $N(a)-\{w\}, N(b)-\{w\} \subseteq N_{2}(w)$. Since $G$ is $K_{2, m}$-free, $|N(a) \cap N(b)| \leqslant m-1$, and hence $|A| \geqslant 2(m-1)^{7}-m+1$ and $|B| \geqslant 2(m-1)^{7}-m+1$. Since $G$ is 3 -connected, $G-w$ is 2-connected. Let $k$ be the maximum order of an induced $a-b$ path in $G-w$.

We consider three cases according to the value of $k$.

Case 1: $k=3$

Note that it follows from the assumption of this case that $N(a) \cup N(b)$ is independent. Since $G-w$ is 2-connected, it also follows that $|(N(a) \cap N(b))-\{w\}| \geqslant 2$. Let $c_{1}, c_{2} \in$ $(N(a) \cap N(b))-\{w\}$ with $c_{1} \neq c_{2}$.

Note that we have $N(x) \cap(N(a) \cup N(b))=\varnothing$ for each $x \in A \cup B$ because $N(a) \cup N(b)$ is independent. Let $A_{0}=\{x \in A \mid N(x) \subseteq N(w)\}$ and $A_{1}=A-A_{0}$. For each $x \in A$, we define $u_{x} \in N(x)$ as follows: if $x \in A_{0}$, let $u_{x} \in N(x)-\{a\}$; if $x \in A_{1}$, let $u_{x} \in$ $N(x)-N(w)$. For $A^{\prime} \subseteq A$, let $U_{A^{\prime}}=\left\{u_{x} \mid x \in A^{\prime}\right\}$. Let $B_{0}=\{y \in B \mid N(y) \subseteq N(w)\}$ and $B_{1}=B-B_{0}$. For each $y \in B$, we define $v_{y} \in N(y)$ as follows: if $y \in B_{0}$, let $v_{y} \in N(y)-\{b\}$; if $y \in B_{1}$, let $v_{y} \in N(y)-N(w)$. For $B^{\prime} \subseteq B$, let $V_{B^{\prime}}=\left\{v_{y} \mid y \in B^{\prime}\right\}$.

Claim 6.1. For $A^{\prime} \subseteq A,\left|U_{A^{\prime}}\right| \geqslant\left|A^{\prime}\right| /(m-1)$. For $B^{\prime} \subseteq B,\left|V_{B^{\prime}}\right| \geqslant\left|B^{\prime}\right| /(m-1)$.

Proof. Let $A^{\prime} \subseteq A$. Suppose that $\left|U_{A^{\prime}}\right|<\left|A^{\prime}\right| /(m-1)$. Then there exists $u \in U_{A^{\prime}}$ such that $u=u_{x}$ for some $m$ vertices $x$ in $A^{\prime}$. Hence $G[\{a, u\} \cup(N(a) \cap N(u))]$ contains $K_{2, m}$ as an induced subgraph, a contradiction. Thus $\left|U_{A^{\prime}}\right| \geqslant\left|A^{\prime}\right| /(m-1)$. Similarly, we get $\left|V_{B^{\prime}}\right| \geqslant\left|B^{\prime}\right| /(m-1)$ for $B^{\prime} \subseteq B$.

We consider two subcases.

Subcase 1.1: $A_{1}=\varnothing$ or $B_{1}=\varnothing$

We may assume that $A_{1}=\varnothing$. By Claim 6.1 and the fact that $\left|A_{0}\right|=|A| \geqslant 2(m-$ $1)^{7}-m+1>(m-1)^{2},\left|U_{A_{0}}\right| \geqslant m$. Since $|B| \geqslant 2(m-1)^{7}-m+1>(m-1)^{2}$, $\left|V_{B}\right| \geqslant m$ by Claim 6.1. Since $G$ is $K_{3}$-free, it follows that if both $c_{1}$ and $c_{2}$ are adjacent 
to all vertices in $V_{B}$, then $G\left[\left\{c_{1}, c_{2}\right\} \cup V_{B}\right]$ contains $K_{2, m}$ as an induced subgraph, a contradiction. Thus $c_{h} v_{y} \notin E(G)$ for some $h \in\{1,2\}$ and some $y \in B$. Then since $v_{y} \notin N(a) \cup N(b), a c_{h} b y v_{y}$ is an induced path. Note that $U_{A_{0}} \subseteq N(w)$. Since $G$ is $K_{2, m^{-}}$ free, $\left|N\left(c_{h}\right) \cap U_{A_{0}}\right| \leqslant\left|N\left(c_{h}\right) \cap N(w)\right| \leqslant m-1$. Since $\left|U_{A_{0}}\right| \geqslant m$, there exists $x \in A_{0}$ such that $u_{x} c_{h} \notin E(G)$. Then $u_{x} x a c_{h} b$ is an induced path. Since $N(a) \cup N(b)$ is independent, $x y \notin E(G)$. Since $k=3$, we also have $u_{x} y, x v_{y}, u_{x} v_{y} \notin E(G)$. Consequently the path $u_{x} x a c_{h} b y v_{y}$ is an induced path of order 7 , a contradiction.

Subcase 1.2: $A_{1} \neq \varnothing$ and $B_{1} \neq \varnothing$

Let $x \in A_{1}$ and $y \in B_{1}$. Then since $u_{x}, v_{y} \notin N(a) \cup N(b) \cup N(w)$ and $k=3, u_{x} x a w b y v_{y}$ is an induced path of order 7 , a contradiction.

Case 2: $k=4$

Let $H_{1}, \ldots, H_{p}$ be the components of $G[A \cup B]$. Let $1 \leqslant i \leqslant p$. Suppose that there exists $x \in V\left(H_{i}\right)$ such that $d_{H_{i}}(x) \geqslant m$. We may assume that $x \in A$. Then $N_{H_{i}}(x) \subseteq B$, which implies that $G\left[\{b\} \cup V\left(H_{i}\right)\right]$ contains $K_{2, m}$ as an induced subgraph, a contradiction. Thus $\Delta\left(H_{i}\right) \leqslant m-1$. Since $H_{i}$ is $P_{7}$-free, we also have $\operatorname{diam}\left(H_{i}\right) \leqslant 5$. Consequently $\left|V\left(H_{i}\right)\right| \leqslant(m-1)^{5}$ for each $1 \leqslant i \leqslant p$. Since $|A \cup B| \geqslant 2\left(2(m-1)^{7}-m+1\right)$, it follows that $p \geqslant 2\left(2(m-1)^{7}-m+1\right) /(m-1)^{5}>3(m-1)^{2}+2$. Since $k=4,\left|V\left(H_{i}\right)\right| \geqslant 2$ for some $i$. We may assume that $\left|V\left(H_{1}\right)\right| \geqslant 2$. Let $a_{1} b_{1} \in E\left(H_{1}\right)$ with $a_{1} \in A$ and $b_{1} \in B$.

Note that each vertex in $N(a) \cap N(b)$ is an isolated vertex in $G[N(a) \cup N(b)]$. Since $G-\{a, b\}$ is connected, this implies that for each $2 \leqslant i \leqslant p$, there exists $x_{i} \in V\left(H_{i}\right)$ such that $N\left(x_{i}\right)-(\{a, b\} \cup N(a) \cup N(b)) \neq \varnothing$. Let $X=\left\{x_{i} \mid 2 \leqslant i \leqslant p\right\}$. Let $A_{0}=\{x \in A \cap X \mid N(x)-(\{a, b\} \cup N(a) \cup N(b)) \subseteq N(w)\}$ and $A_{1}=(A \cap X)-A_{0}$. For each $x \in A \cap X$, we define $u_{x} \in N(x)$ as follows: if $x \in A_{0}$, let $u_{x} \in N(x)-(\{a, b\} \cup N(a) \cup N(b))$; if $x \in A_{1}$, let $u_{x} \in N(x)-(\{a, b\} \cup N(a) \cup N(b))-N(w)$. For $A^{\prime} \subseteq A \cap X$, let $U_{A^{\prime}}=\left\{u_{x} \mid x \in A^{\prime}\right\}$. Let $B_{0}=\{y \in B \cap X \mid N(y)-(\{a, b\} \cup N(a) \cup N(b)) \subseteq N(w)\}$ and $B_{1}=(B \cap X)-B_{0}$. For each $y \in B \cap X$, we define $v_{y} \in N(y)$ as follows: if $y \in B_{0}$, let $v_{y} \in N(y)-(\{a, b\} \cup N(a) \cup N(b))$; if $y \in B_{1}$, let $v_{y} \in N(y)-(\{a, b\} \cup N(a) \cup N(b))-N(w)$. For $B^{\prime} \subseteq B \cap X$, let $V_{B^{\prime}}=\left\{v_{y} \mid y \in B^{\prime}\right\}$. Note that $\left|A_{0}\right|+\left|A_{1}\right|+\left|B_{0}\right|+\left|B_{1}\right|=|X|=p-1$. Arguing as in the proof of Claim 6.1, we obtain the following claim.

Claim 6.2. For $A^{\prime} \subseteq A \cap X,\left|U_{A^{\prime}}\right| \geqslant\left|A^{\prime}\right| /(m-1)$. For $B^{\prime} \subseteq B \cap X,\left|V_{B^{\prime}}\right| \geqslant\left|B^{\prime}\right| /(m-1)$.

Here we consider the following three subcases:

(1) $\left|A_{0}\right|>(m-1)^{2}$ or $\left|B_{0}\right|>(m-1)^{2}$;

(2) $\left|A_{0}\right| \leqslant(m-1)^{2}$ and $\left|B_{0}\right| \leqslant(m-1)^{2}$ and, moreover, we have $A_{1} \neq \varnothing$ and $B_{1} \neq \varnothing$;

(3) $\left|A_{0}\right| \leqslant(m-1)^{2}$ and $\left|B_{0}\right| \leqslant(m-1)^{2}$, but we have $A_{1}=\varnothing$ or $B_{1}=\varnothing$.

Subcase 2.1: $\left|A_{0}\right|>(m-1)^{2}$ or $\left|B_{0}\right|>(m-1)^{2}$

We may assume that $\left|A_{0}\right|>(m-1)^{2}$. By Claim 6.2, $\left|U_{A_{0}}\right| \geqslant m$. Note that $U_{A_{0}} \subseteq$ $N(w)$. Since $G$ is $K_{2, m}$-free, $\left|N\left(a_{1}\right) \cap U_{A_{0}}\right| \leqslant m-1$. Since $\left|U_{A_{0}}\right| \geqslant m$, there exists $x \in A_{0}$ such that $u_{x} a_{1} \notin E(G)$. We may assume that $x=x_{2}$. Since $x_{2}$ and $b_{1}$ belong to distinct components of $G[A \cup B], x_{2} b_{1} \notin E(G)$. Since $|B| \geqslant 2(m-1)^{7}-m+1>2(m-1)^{5} \geqslant$ $\left|V\left(H_{1}\right)\right|+\left|V\left(H_{2}\right)\right|, B-\left(V\left(H_{1}\right) \cup V\left(H_{2}\right)\right) \neq \varnothing$. Let $y \in B-\left(V\left(H_{1}\right) \cup V\left(H_{2}\right)\right)$. Then 
$y a_{1}, y x_{2} \notin E(G)$. Since $k=4$, we also have $u_{x_{2}} b_{1}, u_{x_{2}} y \notin E(G)$. Since $u_{x_{2}} \notin N(a) \cup N(b)$, we now see that the path $u_{x_{2}} x_{2} a a_{1} b_{1} b y$ is an induced path of order 7 , a contradiction.

Subcase 2.2: $\left|A_{0}\right| \leqslant(m-1)^{2}$ and $\left|B_{0}\right| \leqslant(m-1)^{2}$ and, moreover, we have $A_{1} \neq \varnothing$ and $B_{1} \neq \varnothing$

Let $x \in A_{1}$ and $y \in B_{1}$. By the definition of $X, x$ and $y$ belong to distinct components of $G[A \cup B]$, and hence $x y \notin E(G)$. Since $u_{x}, v_{y} \notin N(a) \cup N(b) \cup N(w)$ and $k=4$, it follows that the path $u_{x} x a w b y v_{y}$ is an induced path of order 7 , a contradiction.

Subcase 2.3: $\left|A_{0}\right| \leqslant(m-1)^{2}$ and $\left|B_{0}\right| \leqslant(m-1)^{2}$, but we have $A_{1}=\varnothing$ or $B_{1}=\varnothing$

We may assume that $A_{1}=\varnothing$. Then $3(m-1)^{2}+1<p-1=\left|A_{0}\right|+\left|B_{0}\right|+\left|B_{1}\right| \leqslant$ $\left|B_{1}\right|+2(m-1)^{2}$, and hence $\left|B_{1}\right|>(m-1)^{2}+1$. On the other hand, since $|A| \geqslant$ $2(m-1)^{7}-m+1>(m-1)^{2}(m-1)^{5} \geqslant\left|A_{0}\right|(m-1)^{5}=\left|A_{0} \cup A_{1}\right|(m-1)^{5}=|A \cap X|(m-1)^{5}$ and since $\left|V\left(H_{i}\right)\right| \leqslant(m-1)^{5}$ for each $2 \leqslant i \leqslant p$, there exists $i$ with $2 \leqslant i \leqslant p$ such that $V\left(H_{i}\right) \cap A \neq \varnothing$ and $x_{i} \notin A$. We may assume that $V\left(H_{2}\right) \cap A \neq \varnothing$ and $x_{2} \notin A$. Then $x_{2} \in B$, which implies $\left|V\left(H_{2}\right)\right| \geqslant 2$. Let $a_{2} b_{2} \in E\left(H_{2}\right)$ with $a_{2} \in A$ and $b_{2} \in B$. Now since $\left|B_{1}-\left\{x_{2}\right\}\right|>(m-1)^{2},\left|V_{B_{1}-\left\{x_{2}\right\}}\right| \geqslant m$ by Claim 6 .2. Since $G$ is $K_{3}$-free, it follows that if both $b_{1}$ and $b_{2}$ are adjacent to all vertices in $V_{B_{1}-\left\{x_{2}\right\}}$, then $G\left[\left\{b_{1}, b_{2}\right\} \cup V_{B_{1}-\left\{x_{2}\right\}}\right]$ contains $K_{2, m}$ as an induced subgraph, a contradiction. Thus $b_{h} v_{y} \notin E(G)$ for some $h \in\{1,2\}$ and some $y \in B_{1}-\left\{x_{2}\right\}$. We may assume that $y=x_{3}$. Since $|A| \geqslant 2(m-1)^{7}-m+1>$ $3(m-1)^{5} \geqslant\left|V\left(H_{1}\right)\right|+\left|V\left(H_{2}\right)\right|+\left|V\left(H_{3}\right)\right|, A-\left(V\left(H_{1}\right) \cup V\left(H_{2}\right) \cup V\left(H_{3}\right)\right) \neq \varnothing$. Let $x \in A-\left(V\left(H_{1}\right) \cup V\left(H_{2}\right) \cup V\left(H_{3}\right)\right)$. Since $v_{x_{3}} \notin N(a) \cup N(b)$ and $k=4$, the path $x a a_{h} b_{h} b x_{3} v_{x_{3}}$ is an induced path of order 7 , a contradiction.

Case 3: $k \geqslant 5$

If $k=5$, then by Lemma 6.2 and the assumption that $d(a) \geqslant 2(m-1)^{7} \geqslant 4(m-$ $1)+2$ and $d(b) \geqslant 2(m-1)^{7} \geqslant 4(m-1)+2, G$ contains $P_{7}$ as an induced subgraph, a contradiction; if $k \geqslant 6$, then by Lemma 6.1 and the assumption that $d(a) \geqslant 2(m-1)^{7} \geqslant$ $4(m-1)+2, G$ contains $P_{7}$ as an induced subgraph, a contradiction.

This completes the proof of Lemma 6.5.

Proposition 6.6. Let $m \geqslant 5$. Then $\mathcal{G}_{3}\left(\left\{K_{3}, K_{2, m}, P_{7}\right\}\right)$ is finite.

Proof. Let $G \in \mathcal{G}_{3}\left(\left\{K_{3}, K_{2, m}, P_{7}\right\}\right)$. We show that $|V(G)| \leqslant\left(4(m-1)^{7}\left(2(m-1)^{7}+m-\right.\right.$ $\left.2)^{5}\right)^{5}$. Since $\operatorname{diam}(G) \leqslant 5$, it suffices to show that $\Delta(G) \leqslant 4(m-1)^{7}\left(2(m-1)^{7}+m-2\right)^{5}$. Suppose that $\Delta(G) \geqslant 4(m-1)^{7}\left(2(m-1)^{7}+m-2\right)^{5}+1$, and let $w \in V(G)$ be a vertex with $d(w)=\Delta(G)$. Since $G$ is $K_{3}$-free, $N(w)$ is independent. Let $L=\{x \in V(G)$ $\left.\left|N(x) \cap N_{2}(w)\right| \geqslant 2(m-1)^{7}\right\}$. By Lemma 6.5, $|L \cap N(w)| \leqslant 1$ and $L \cap N_{2}(w)=\varnothing$.

Case 1: $|L| \geqslant 2$ and $|L \cap N(w)|=1$

Let $a \in L \cap N(w)$ and $b \in L-N(w)$. Since $b \notin N(w) \cup N_{2}(w)$, we have $b \in N_{3}(w)$, and hence $a b \notin E(G)$. By Lemma 6.5, $N(a) \cap N(b)=\varnothing$. Let $y \in N(b) \cap N_{2}(w)$ and $v \in N(y) \cap N(w)$. Since $a b \notin E(G), N(a) \cap N(b)=\varnothing$, and $N(w)$ is independent, it follows that the path awvyb is an induced path of order 5 . Hence by Lemma 6.2 and the assumption that $d(a) \geqslant 2(m-1)^{7} \geqslant 4(m-1)+2$ and $d(b) \geqslant 2(m-1)^{7} \geqslant 4(m-1)+2$, $G$ contains $P_{7}$ as an induced subgraph, a contradiction. 
Case 2: $|L| \geqslant 2$ and $L \cap N(w)=\varnothing$

Let $a, b \in L$ with $a \neq b$. Then $a, b \in N_{3}(w)$. If $a b \in E(G)$, then $N(a) \cap N(b)=\varnothing$ by the assumption that $G$ is $K_{3}$-free and, if $a b \notin E(G)$, then $N(a) \cap N(b)=\varnothing$ by Lemma 6.5. Hence in either case, we have $N(a) \cap N(b)=\varnothing$. Let $A=N(a) \cap N_{2}(w)$ and $B=N(b) \cap N_{2}(w)$. Let $y \in B$ and $v \in N(y) \cap N(w)$. Since $G$ is $K_{2, m}$-free, $|N(y) \cap N(w)| \leqslant m-1$. Since $G$ is $K_{2, m}$-free, it follows that $\mid\left(\left(\bigcup_{u \in N(y) \cap N(w)} N(u)\right) \cup\right.$ $N(y)) \cap A \mid \leqslant(|N(y) \cap N(w)|+1)(m-1) \leqslant m(m-1)$. Since $|A| \geqslant 2(m-1)^{7} \geqslant m(m-1)+1$, $A-\left(\left(\bigcup_{u \in N(y) \cap N(w)} N(u)\right) \cup N(y)\right) \neq \varnothing$. Take $x \in A-\left(\left(\bigcup_{u \in N(y) \cap N(w)} N(u)\right) \cup N(y)\right)$. Since $v \in N(y) \cap N(w)$, we have $x v, x y \notin E(G)$. Take $u \in N(x) \cap N(w)$. By the choice of $x, u \notin$ $N(y) \cap N(w)$, and hence $u y \notin E(G)$. Consequently the path xuwvyb is an induced path of order 6 . Now by Lemma 6.1 and the assumption that $d(b) \geqslant 2(m-1)^{7} \geqslant 4(m-1)+2$, $G$ contains $P_{7}$ as an induced subgraph, a contradiction.

Case 3: $|L| \leqslant 1$

Let $H_{1}, \ldots, H_{p}$ be the components of $G\left[\left(N(w) \cup N_{2}(w)\right)-L\right]$. Let $1 \leqslant i \leqslant p$. Take $x \in V\left(H_{i}\right)$. Since $G$ is $\left\{K_{3}, K_{2, m}\right\}$-free, $|N(x) \cap N(w)| \leqslant m-1$. Since $x \notin L$, we also have $\left|N(x) \cap N_{2}(w)\right| \leqslant 2(m-1)^{7}-1$. Hence $d_{H_{i}}(x) \leqslant|N(x) \cap N(w)|+\left|N(x) \cap N_{2}(w)\right| \leqslant$ $2(m-1)^{7}+m-2$. Since $x \in V\left(H_{i}\right)$ is arbitrary, $\Delta\left(H_{i}\right) \leqslant 2(m-1)^{7}+m-2$. Since $H_{i}$ is $P_{7}$-free, we also have $\operatorname{diam}\left(H_{i}\right) \leqslant 5$. Thus $\left|V\left(H_{i}\right)\right| \leqslant\left(2(m-1)^{7}+m-2\right)^{5}$ for each $1 \leqslant i \leqslant p$. We have $V\left(H_{i}\right) \cap N_{2}(w) \neq \varnothing$ for each $1 \leqslant i \leqslant p$ because $N(w)$ is independent. Let $q=\left|\left\{i \mid V\left(H_{i}\right) \cap N(w) \neq \varnothing\right\}\right|$. (Note that in the case where $\varnothing \neq L \subseteq N(w)$, it is possible that $V\left(H_{i}\right) \cap N(w)=\varnothing$ for some $i$.) Without loss of generality, we may assume that $V\left(H_{i}\right) \cap N(w) \neq \varnothing$ for each $1 \leqslant i \leqslant q$ and $V\left(H_{i}\right) \cap N(w)=\varnothing$ for each $q+1 \leqslant i \leqslant p$. Since $|N(w)-L| \geqslant d(w)-1 \geqslant 4(m-1)^{7}\left(2(m-1)^{7}+m-2\right)^{5}$, we have $q \geqslant|N(w)-L| /\left(2(m-1)^{7}+m-2\right)^{5} \geqslant 4(m-1)^{7}$. Since $G$ is 3-connected, $G-(\{w\} \cup L)$ is connected. Let $P$ be a shortest $V\left(H_{1}\right)-\left(\left(N(w) \cup N_{2}(w)\right)-L-V\left(H_{1}\right)\right)$ path in $G-(\{w\} \cup L)$. Then $|V(P)| \geqslant 3$. Let $y_{1}, z_{1}$ and $z_{2}$ be the first three vertices of $P$. Then $y_{1} \in V\left(H_{1}\right) \cap N_{2}(w)$ and $z_{1} \in N_{3}(w)$. Let $w x_{1} y_{1}$ be a shortest $w-y_{1}$ path in $G\left[V\left(H_{1}\right) \cup\{w\}\right]$. Then $w x_{1} y_{1} z_{1} z_{2}$ is an induced $w-z_{2}$ path of order 5. Since $z_{1} \notin L$, $\left|N\left(z_{1}\right) \cap N_{2}(w)\right| \leqslant 2(m-1)^{7}-1$. In particular, $z_{1}$ is adjacent to at most $2(m-1)^{7}-1$ of the $H_{i}, 1 \leqslant i \leqslant q$. If $z_{2} \notin N_{2}(w)$, then similarly, $z_{2}$ is adjacent to at most $2(m-1)^{7}-1$ of the $H_{i}, 1 \leqslant i \leqslant q$; if $z_{2} \in N_{2}(w)$, then clearly $z_{2}$ is adjacent to at most one of the $H_{i}$, $1 \leqslant i \leqslant q$ (note that it is possible that $z_{2}$ belongs to $H_{i}$ for some $i$ with $q+1 \leqslant i \leqslant p$ ). Thus $z_{2}$ is adjacent to at most $2(m-1)^{7}-1$ of the $H_{i}$. Since $q \geqslant 4(m-1)^{7}$, there exists $j$ with $2 \leqslant j \leqslant q$ such that neither $z_{1}$ nor $z_{2}$ is adjacent to $H_{j}$. Since $V\left(H_{j}\right) \cap N(w) \neq \varnothing$ and $V\left(H_{j}\right) \cap N_{2}(w) \neq \varnothing$, there exist $x_{2} \in V\left(H_{j}\right) \cap N(w)$ and $y_{2} \in V\left(H_{j}\right) \cap N_{2}(w)$ such that $x_{2} y_{2} \in E(G)$. Then $y_{2} x_{2} w x_{1} y_{1} z_{1} z_{2}$ is an induced path of order 7 , a contradiction.

This completes the proof of Proposition 6.6.

Proof of Theorem 1.4. The 'if' part follows from Propositions 6.4 and 6.6. Thus it suffices to prove the 'only if' part. Suppose that $\mathcal{G}_{3}\left(\left\{K_{3}, K_{2, m}, T\right\}\right)$ is finite. From Theorem 1.1, it follows that $T \in \mathcal{T}_{2}$. For each $s \geqslant 2$, let $H_{s}^{(1)}$ be the graph defined by $V\left(H_{s}^{(1)}\right)=$ $\left\{x_{1}, x_{2}, x_{3}\right\} \cup\left\{y_{i, h}, z_{i, h} \mid 1 \leqslant i \leqslant 2,1 \leqslant h \leqslant s\right\}$ and $E\left(H_{s}^{(1)}\right)=\left\{x_{1} x_{2}\right\} \cup\left\{x_{i} y_{i, h}, x_{3} z_{i, h} \mid\right.$ $1 \leqslant i \leqslant 2,1 \leqslant h \leqslant s\} \cup\left\{y_{i, h} z_{j, h} \mid 1 \leqslant i, j \leqslant 2,1 \leqslant h \leqslant s\right\}$ (see Figure 6). Then $H_{s}^{(1)}$ is 
3-connected and $\left\{K_{3}, K_{2, m}\right\}$-free. Since $\mathcal{G}_{3}\left(\left\{K_{3}, K_{2, m}, T\right\}\right)$ is finite, there exists $s \geqslant 2$ such that $H_{s}^{(1)}$ contains $T$ as an induced subgraph. Since every tree contained in $H_{s}^{(1)}$ as an induced subgraph has diameter at most $6, \operatorname{diam}(T) \leqslant 6$. Now for each $t \geqslant 3$, let $P=x_{1} x_{2}$ be a path of order 2 and $C=y_{1} y_{2} \cdots y_{2 t} y_{1}$ be a cycle of order $2 t$, and let $H_{t}^{(2)}$ be the graph defined by $V\left(H_{t}^{(2)}\right)=V(C) \cup V(P)$ and $E\left(H_{t}^{(2)}\right)=E(C) \cup E(P) \cup\left\{x_{1} y_{2 i-1}, x_{2} y_{2 i} \mid\right.$ $1 \leqslant i \leqslant t\}$ (see Figure 6). Then $H_{t}^{(2)}$ is 3 -connected and $\left\{K_{3}, K_{2, m}\right\}$-free. Hence there exists $t \geqslant 3$ such that $H_{t}^{(2)}$ contains $T$ as an induced subgraph. Observe that if $F$ is a member of $\mathcal{T}_{2}$ contained in $H_{t}^{(2)}$ as an induced subgraph, then $F$ is a path or an induced subgraph of $Y_{3}^{*}$. Therefore $T$ is either a path of order at most 7 or an induced subgraph of $Y_{3}^{*}$.
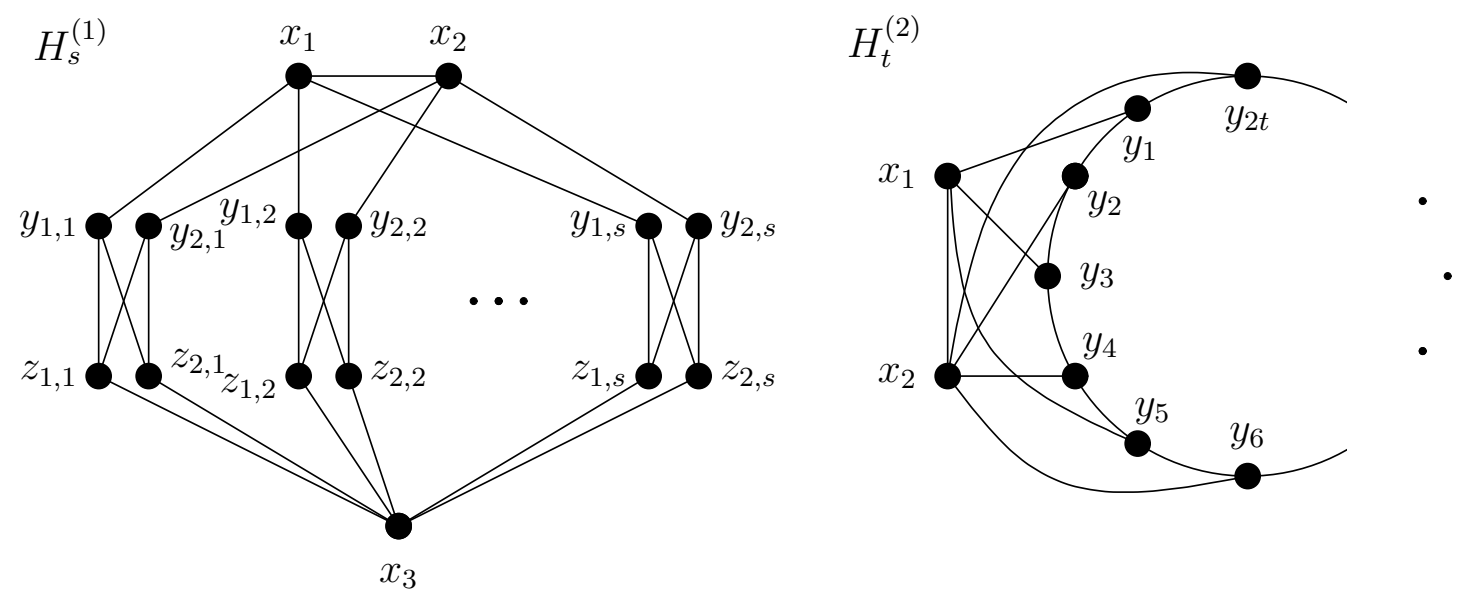

Figure 6: Graphs $H_{s}^{(1)}$ and $H_{t}^{(2)}$

\section{Concluding remarks}

In this paper, we have considered three forbidden subgraphs which generate a finite set in the class of 3-connected graphs. As we have seen in Theorem 1.1, there are six types. For many of them, we have given a characterization. The cases which remain uncharacterized are the following:

(a) $\mathcal{F}=\left\{K_{3}, K_{3,3}, T\right\}$, where $T \in \mathcal{T}_{2}$;

(b) $\mathcal{F}=\left\{K_{4}, K_{2,2}, T\right\}$, where $T$ is a path of order at least 4;

(c) $\mathcal{F}=\left\{K_{3}, K_{2,4}, T\right\}$ and $\left\{K_{3}, K_{2,3}, T\right\}$, where $T \in \mathcal{T}_{1}$;

(d) $\mathcal{F}=\left\{K_{3}, K_{2,2}, T\right\}$, where $T \in \mathcal{T}_{0}$;

(e) $\mathcal{F}=\left\{K_{3}, K_{1,4}, T\right\}$, where $T \in \mathcal{T}_{1}$;

(f) $\mathcal{F}=\left\{K_{n}, K_{1,3}, T\right\}$, where $n \geqslant 5$ and $T \in \mathcal{T}_{2}^{*}$;

(g) $\mathcal{F}=\left\{K_{4}, K_{1,3}, T\right\}$, where $T \in \mathcal{T}_{1}^{*}$.

In cases $(\mathrm{a})-(\mathrm{d}), \mathcal{F}$ does not contain a star. In these cases, we can give a bound on the diameter of $T$. 
Proposition 7.1. Let $\mathcal{F}$ be a subset of $\mathcal{G}$ with $|\mathcal{F}|=3$. Suppose $\mathcal{F}$ does not contain a star and $\mathcal{G}_{3}(\mathcal{F})$ is finite. Then $\mathcal{F}$ contains a tree of diameter at most 8.

Proof. Since $\mathcal{F}$ does not contain a star, it follows from Theorem 1.1 that $\mathcal{F}$ can be written in the form $\mathcal{F}=\left\{K_{n}, K_{m_{1}, m_{2}}, T\right\}$, where $n \in\{3,4\}, m_{1} \in\{2,3\}, m_{2} \geqslant m_{1}$ and $T \in \mathcal{T}_{0}$.

Let $s \geqslant 2$. Let $C^{(i)}=a_{1}^{(i)} b_{1}^{(i)} c_{1}^{(i)} a_{2}^{(i)} b_{2}^{(i)} c_{2}^{(i)} a_{1}^{(i)}$ be a cycle of order 6 for each $1 \leqslant$ $i \leqslant s$, and define a graph $H_{s}$ by $V\left(H_{s}\right)=\left\{a_{0}, b_{0}, c_{0}\right\} \cup\left(\bigcup_{1 \leqslant i \leqslant s} V\left(C^{(i)}\right)\right)$ and $E\left(H_{s}\right)=$ $\left\{a_{0} a_{j}^{(i)}, b_{0} b_{j}^{(i)}, c_{0} c_{j}^{(i)} \mid 1 \leqslant i \leqslant s, 1 \leqslant j \leqslant 2\right\} \cup\left(\bigcup_{1 \leqslant i \leqslant s} E\left(C^{(i)}\right)\right)$. Then $H_{s}$ is 3-connected and $\left\{K_{n}, K_{m_{1}, m_{2}}\right\}$-free. Hence there exists $s \geqslant 2$ such that $H_{s}$ contains $T$ as an induced subgraph. However, $H_{s}$ does not contain $P_{10}$ as an induced subgraph. Therefore the diameter of $T$ is at most 8 .

In (a)-(d), we have $T \in \mathcal{T}_{0}$ and hence $\Delta(T) \leqslant 3$. By combining this fact and Proposition 7.1, we see that the order of $T$ is bounded. Thus the number of triples in these cases is finite. On the other hand, in cases $(\mathrm{e})-(\mathrm{g})$, where the triple contains a star, Proposition 7.1 gives no further information about $\mathcal{F}$. In fact, Theorem A shows that in these cases, there exist infinitely many $\mathcal{F}$ such that $\mathcal{G}_{3}(\mathcal{F})$ is finite.

We add that for (a) and (b), and for the case where $\mathcal{F}=\left\{K_{3}, K_{2,4}, T\right\}$ in (c), the determination of $T$ has recently been completed in [2].

\section{Acknowledgments}

The authors are grateful to the referees for helpful comments. In particular, one of them has pointed out errors in the first version of the paper.

\section{References}

[1] R. Diestel. Graph Theory (4th edition). Graduate Texts in Mathematics 173, Springer, 2010.

[2] Y. Egawa and M. Furuya. Forbidden triples containing a complete graph and a complete bipartite graph of small order. Graphs Combin. 30 (2014) 1149-1162.

[3] J. Fujisawa, M.D. Plummer and A. Saito. Forbidden subgraphs generating a finite set. Discrete Math. 313 (2013) 1835-1842.

[4] M. Kochol. Snarks without small cycles. J. Combin. Theory Ser. B 67 (1996) 34-47. 